\title{
Multiport based teleportation - transmission of a large amount of quantum information
}

\author{
Piotr Kopszak ${ }^{1}$, Marek Mozrzymas ${ }^{1}$, Michał Studziński ${ }^{2}$ and Michał Horodecki ${ }^{3}$ \\ 1 Institute for Theoretical Physics, University of Wrocław 50-204 Wrocław, Poland \\ 2 Institute of Theoretical Physics and Astrophysics and National Quantum Information Centre in Gdańsk, Faculty of Mathematics, Physics \\ and Informatics, University of Gdańsk, 80-952 Gdańsk, Poland \\ 3 International Centre for Theory of Quantum Technologies, University of Gdańsk, 80-952, Poland
}

We analyse the problem of transmitting a number of unknown quantum states or one composite system in one go. We derive a lower bound on the performance of such process, measured in the entanglement fidelity. The obtained bound is effectively computable and outperforms the explicit values of the entanglement fidelity calculated for the pre-existing variants of the port-based protocols, allowing for teleportation of a much larger amount of quantum information. The comparison with the exact formulas and similar analysis for the probabilistic scheme is also discussed. In particular, we present the closed-form expressions for the entanglement fidelity and for the probability of success in the probabilistic scheme in the qubit case in the picture of the spin angular momentum.

\section{Introduction}

In 2008 the novel port-based teleportation protocol (PBT) has been proposed [14, 15]. In contrast to the very first teleportation procedure, discovered in [5], it does not require a correction on the receiver's side depending on the classical outcome of the sender's measurement, see Figure 1. The lack of the correction led to various new applications, where the ordinary teleportation fails, like for example NISQ protocols [3, 14], position-based cryptography [4], fundamental limitations on quantum channels discrimination [24], connection between non-locality and complexity [7], and many other important results $[8,16,21,23,25,27]$.

The huge advantage of the lack of receiver's correction comes at a price. Due to no-programming theorem [22] the ideal transmission in such scheme is possible only when parties exploit an infinite number of maximally entangled pairs. Accordingly, we distinguish deterministic scenario where teleportation is imperfect and the state after teleportation is distorted and probabilistic scenario where teleportation is perfect but one has to accept the non-zero failure probability of the whole process. In the first case, to learn

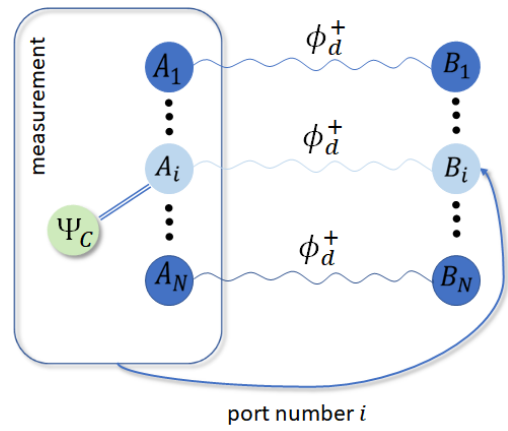

Figure 1: The standard configuration for the PBT scheme: two parties share $N$ copies of the maximally entangled state, called port, $\phi_{d}^{+}=\left|\phi_{d}^{+}\right\rangle\left\langle\phi_{d}^{+}\right|$, where $\left|\phi_{d}^{+}\right\rangle=(1 / \sqrt{d}) \sum_{i}|i i\rangle$. Alice (sender) to send an unknown state $\Psi_{C}$ to Bob (receiver), performs a global measurement (POVM) on the states and her half of the maximally entangled pairs. As an output she gets a classical output $1 \leq i \leq N$ indicating ports on the Bob's side where the state arrives. To recover the state Bob has only to pick up the right port, according to the classical message $i$ obtained from Alice, no further correction is needed. We have also the optimised version of PBT, where Alice optimises jointly measurements and the shared states with Bob before she runs the protocol.

about the efficiency we ask how the fidelity of the teleported particle depends on the number of shared entangled pairs, while in the latter we ask about the similar dependence for probability of success of perfect transmission. We can calculate the performance of the non-optimal PBT, where parties share maximally entangled pairs, and optimised PBT, where Alice optimises jointly over the shared state and measurements before she runs the protocol, see Figure 1.

We rigorously address here a fundamental question of transmission capability of PBT raised firstly in [28]. Namely we ask: how many qubits can one faithfully teleport for a given number of ports?

To perform teleportation of a state of $k$ multiparty systems one can: use the PBT with port dimension large enough, apply the PBT several times (sequential PBT), divide total number of ports into smaller packages and send every particle via such subsystem (packaged PBT), or finally apply multi-port based tele- 
portation (MPBT) suggested in [28]. In particular, one could also run the recycling protocol for PBT suggested in [28], however due to recent results derived in [31] the efficiency of such scheme is an open problem. In the first case, increasing dimensionality of the port significantly reduces the entanglement fidelity [15, 18, 19, 29], requiring increasing the number of used ports to compensate it [9]. In sequential PBT and recycling protocol, we have to resign from singleshot scenario and after each round one needs to store the transmitted state, or we have to use distorted resource state, affecting the performance. In MPBT we allow to teleport a quantum multiparty state in one go, where each subsystem of the teleported multiparty system ends up in one of Bob's ports, pointed by Alice's message (see Fig. 2). The protocol thus enjoys quite a mild correction: Bob has to permute ports according to Alice's message.

The main purpose of this paper is to evaluate and compare the transmission capability of PBT and MPBT schemes. More precisely, we consider teleportation of $\sim N^{\alpha}$ qubits (or more generally qudits) through $N$ ports, where $N$ grows to infinity, and analyse the quality of transmission, i.e. we ask about fidelity or probability of success. Note, in contrast with the traditional approach to channel capacity, where the central quantity is the asymptotic rate $k / N$, here the main objective becomes to identify asymptotic exponents $\alpha$ for which the transmission is possible, since the mentioned rate vanishes for port based teleportation.

In any variant of either of PBT and MPBT we obtain critical-like behaviour of the quality of transmission. We identify the critical values $\alpha_{\mathrm{cr}}$ of exponents $\alpha$ for several variants, both for exact asymptotic values of a figure of merit and their lower bounds. Whenever the value of $\alpha$ is below the critical value $\alpha_{\text {cr }}$ the values of fidelity (or probability of success, depending on the scheme) describing transmission are 1 , and 0 otherwise.

We obtain qualitative difference between deterministic scheme and probabilistic one. Namely, in the deterministic scheme, even the optimal PBT scheme is outperformed by non-optimal MPBT (i.e. one based on shared maximally entangled pairs). In particular, we argue that in non-optimal deterministic MPBT, one can teleport a much larger amount of quantum information, i.e. with $\alpha_{\mathrm{cr}}=1$, in comparison to optimal port-based teleportation, where $\alpha_{\mathrm{cr}}=2 / 3$, and one can teleport faithfully only up to $o\left(N^{2 / 3}\right)$ qubits.

Unlike in deterministic variant in probabilistic nonoptimal MPBT the scaling is the same as for probabilistic optimal PBT, allowing for teleportation $o\left(N^{\alpha}\right)$ qubits with $\alpha_{\text {cr }}=1 / 2$. However, considering an optimal version of probabilistic MPBT, one can transmit $o\left(N^{\alpha}\right)$ with a critical exponent equal to 1 , clearly outperforming $\mathrm{PBT}$ variants.

To achieve our results, we first provide a lower bound on fidelity in deterministic non-optimal MPBT based on the state discrimination problem. The new bound is effectively computable and depends only on global parameters like the number of ports, their dimension, and the number of teleported particles. The first bound of such kind has been discussed in [28] in not fully rigorous way, suffering from some flaws discussed later in this paper. In the qubit case, starting from group-theoretical results for exact values of entanglement fidelity and probability of success in nonoptimal MPBT, presented in companion paper [30], we deliver exact and effectively computable expressions for these quantities in qubit case, phrased in appealing form of spin angular momentum. Next, result regarding asymptotic behaviour of non-optimal probabilistic case, have been obtained by combining advanced tools from statistical analysis, in particular non-straightforwardly the celebrated Berry-Essen theorem $[6,10]$ with direct estimates of binomial expressions by their Gaussian approximations. For optimal probabilistic MPBT we use for our analysis the exact formula for probability of success in such optimal MPBT derived in [20]. In the Table 1 we summarise the already mentioned variations of the architecture of PBT protocols, which are studied in this paper and are presented in more detail in the following sections.

\section{The Multi-port-based Teleportation}

In order to transmit $k$-system state $\Psi_{C}=$ $\Psi_{C_{1} C_{2} \ldots C_{k}}$ (see Fig. 2) Alice applies a global measurement $\Pi_{i}^{A C}$, where $A=A_{1} \ldots A_{N}$, on her halves of state $\Phi_{A B}^{+}=\bigotimes_{j=1}^{N}\left|\phi_{d}^{+}\right\rangle\left\langle\left.\phi_{d}^{+}\right|_{A_{j} B_{j}}\right.$ and the state to be teleported. As an output she receives a tuple of indices $\mathbf{i}=\left\{i_{1}, i_{2}, \ldots, i_{k}\right\}$ and sends it to Bob through a classical channel. We denote the set of all outputs i by $\mathcal{I}$. Note that the number of outputs $k !\left(\begin{array}{c}N \\ k\end{array}\right)$ grows polynomially in $N$ at fixed $k$. The meaning of indices $\left(i_{1}, \ldots, i_{k}\right)$ is that they point the ports on the receiver's side on which the teleported systems appear, i.e. the first system arrives at port $i_{1}$, the second at $i_{2}$ and so on. Bob recovers the initial form of the teleported state by suitably permuting his systems. The resulting action of the teleportation channel $\mathcal{N}$ is of the form

$$
\begin{aligned}
& \mathcal{N}\left(\Psi_{C}\right)=\sum_{\mathbf{i} \in \mathcal{I}} \operatorname{Tr}_{A \bar{B}_{\mathbf{i}} C}\left[\sqrt{\Pi_{\mathbf{i}}^{A C}}\left(\Phi_{A B}^{+} \otimes \Psi_{C}\right){\sqrt{\Pi_{\mathbf{i}}^{A C}}}^{\dagger}\right] \\
& =\sum_{\mathbf{i} \in \mathcal{I}} \operatorname{Tr}_{A C}\left[\Pi_{\mathbf{i}}^{A C}\left(\sigma_{\mathbf{i}}^{A B} \otimes \Psi_{C}\right)\right],
\end{aligned}
$$

where the bar in $\bar{B}_{\mathbf{i}}$ denotes discarded subsystems except those on positions $i_{1}, i_{2}, \ldots, i_{k}$. The states $\sigma_{\mathbf{i}}^{A B}$ or shortly $\sigma_{\mathbf{i}}$ for $\mathbf{i} \in \mathcal{I}$, called later the signals, are given as

$$
\sigma_{\mathbf{i}}^{A B} \equiv \operatorname{Tr}_{\bar{B}_{\mathbf{i}}} \Phi_{A B}^{+}=\frac{1}{d^{N-k}} \mathbf{1}_{\bar{A}_{\mathbf{i}}} \otimes \phi_{A_{\mathbf{i}} B_{\mathbf{i}}}^{+},
$$




\begin{tabular}{|c|c|c|c|}
\hline Acronym & Name & Description & Reference \\
\hline$(O) P B T$ & $\begin{array}{l}\text { (Optimal) } \\
\text { port- } \\
\text { based } \\
\text { teleportation }\end{array}$ & $\begin{array}{l}\text { Teleportation of } \\
\text { one qudit using } \\
N \text { entangled } \\
\text { pairs, possibly } \\
\text { with optimiza- } \\
\text { tion over states } \\
\text { and POVMs em- } \\
\text { ployed (optimal } \\
\text { protocol) }\end{array}$ & $\begin{array}{l}\text { qbit } \\
\text { case: } \\
{[14,15]} \\
\text { qdit } \\
\text { case:[19, } \\
29]\end{array}$ \\
\hline$(O) M P$ & $\begin{array}{l}T \text { (Optimal) } \\
\text { multi } \\
\text { port- } \\
\text { based } \\
\text { teleportatior }\end{array}$ & $\begin{array}{l}\text { The teleporta- } \\
\text { tion of } k \text { qudits } \\
\text { in one go using } \\
N \text { entangled- } \\
\text { i pairs, includes } \\
\text { the correction } \\
\text { procedure in the } \\
\text { last step, namely } \\
\text { the permutation } \\
\text { of the ports, see } \\
\text { Fig. } 2\end{array}$ & $\begin{array}{l}\text { this } \\
\text { paper; } \\
\text { com- } \\
\text { panion } \\
\text { papers } \\
{[20,30]}\end{array}$ \\
\hline $\begin{array}{l}\text { Pack. } \\
(O) P B T\end{array}$ & $\begin{array}{l}\text { Packaged } \\
\text { (optimal) } \\
\text { port- } \\
\text { based } \\
\text { teleportatiol }\end{array}$ & $\begin{array}{l}\text { Teleportation } \\
\text { of } k \text { qdits by } \\
\text { using } k(\mathrm{O}) \mathrm{PBT} \\
\text { schemes con- } \\
\text { sisting of } N / k \\
\text { entangled-pairs, } \\
\text { see Fig. } 3\end{array}$ & {$[28]$} \\
\hline
\end{tabular}

Table 1: Summary of different variations of Port Based Teleportation protocols discussed in this work. In each of them both deterministic and probabilistic scheme can be realised by choosing the appropriate measurement.

where $\phi_{A_{\mathbf{i}} B_{\mathbf{i}}}^{+}=\left|\phi_{d}^{+}\right\rangle\left\langle\left.\phi_{d}^{+}\right|_{A_{\mathbf{i}} B_{\mathrm{i}}}\right.$. To examine the efficiency we compute the entanglement fidelity which reports how well parties can transmit quantum correlations by sending $k$ halves of maximally entangled states $\Phi_{C D}^{+}=\phi_{C_{1} D_{1}}^{+} \otimes \phi_{C_{2} D_{2}}^{+} \otimes \cdots \otimes \phi_{C_{k} D_{k}}^{+}$:

$F=\operatorname{Tr}\left[\Phi_{B D}^{+}\left(\mathcal{N} \otimes \mathbf{1}_{D}\right)\left(\Phi_{C D}^{+}\right)\right]=\frac{1}{d^{2 k}} \sum_{\mathbf{i} \in \mathcal{I}} \operatorname{Tr}\left[\Pi_{\mathbf{i}}^{A B} \sigma_{\mathbf{i}}^{A B}\right]$

In the probabilistic scheme the teleportation channel looks exactly as in (1), but with different form of measurements. The channel is now trace non-preserving, since there is a POVM, denoted as $\Pi_{\mathbf{0}^{A B}}$, corresponding to the failure of the whole process. The average probability of success reads [14, 30]:

$$
p_{\text {succ }}=\frac{1}{d^{N+k}} \sum_{\mathbf{i} \in \mathcal{I}} \operatorname{Tr}\left[\Pi_{\mathbf{i}}^{A B}\right] .
$$

The goal here is to find the set of optimal measurements, maximising the probability of success. This is done in [30] by exploiting symmetries exhibit in the problem and methods from semidefinite programming. Our considerations look similar to the original

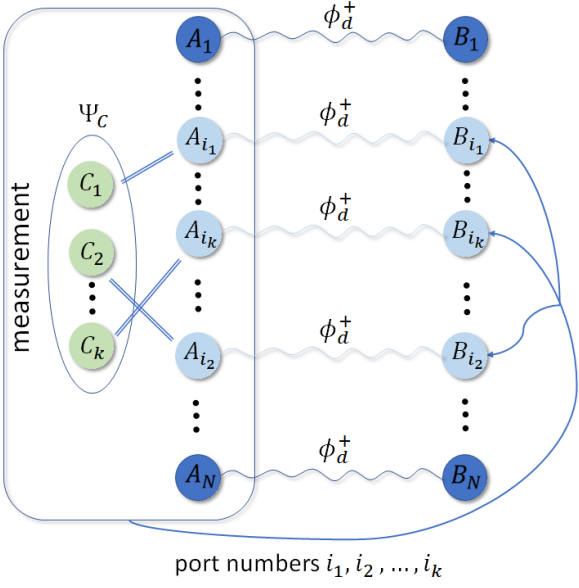

Figure 2: In MPBT two parties share $N$ copies of the maximally entangled state (port) $\phi_{d}^{+}=\left|\phi_{d}^{+}\right\rangle\left\langle\phi_{d}^{+}\right|$, where $\left|\phi_{d}^{+}\right\rangle=$ $(1 / \sqrt{d}) \sum_{i}|i i\rangle$. Alice to transmit a multipartite state $\theta_{C}$ of $k$ systems performs a global measurement (POVM) on the state $\Psi_{C}$ and her halves of the states $\phi_{d}^{+}$. As an output she gets a string $\mathbf{i}=\left\{i_{1}, i_{2}, \ldots, i_{k}\right\}$ indicating ports on the Bob's side where the states arrive. To recover the state Bob has to pick up pointed ports in the right order, which is equivalent to permuting the ports according to message i. Similarly as it is for PBT here Alice can also jointly optimise over shared states and measurements getting optimal MPBT protocol.

PBT scheme, but incorporating $k$ particles into the teleportation process makes it structurally entirely different, see [30]. The structure of the signals $\sigma_{\mathbf{i}}^{A B}$ and the suitable measurements $\Pi_{\mathbf{i}}^{A B}$, makes the problem of evaluating $F$ and $p_{\text {succ }}$ very hard and technical. Therefore, in the next section, we present an effectively computable lower bound on the entanglement fidelity.

\section{Fidelity bound from the state dis- crimination task}

Evaluation of fidelity in the standard PBT was a formidable task, requiring machinery of representation theory of $S U(2)^{\otimes N}$ for qubits, and much more advanced representation theoretic tools for $d>2$. The resulting formulas are usually not very transparent, expressed in terms of complicated sums of representation theory parameters, for which no explicit expressions are known beyond qubits.

The first attempt to derive the efficiency of deterministic MPBT has been presented in [28]. The combinatorial argumentation used by the authors was incorrect, neglecting deeper complexity of the problem. However, one can save part of argumentation and show that the bound given in [28] is indeed a legitimate lower bound for fidelity, see discussion after example 14 in Appendix B. Here, we go further and give a simple and stronger lower bound for fidelity of the protocol. The starting point is the idea pre- 
sented [14, 15], exploited in [4, 28], of providing lower bound for the standard PBT protocol by relating fidelity of teleportation to probability of success in state discrimination. Let us emphasize, that generalizing this approach to the multiport scheme case requires solving a complex combinatorial problem, contained in Lemma 1.

The relation between the entanglement fidelity and the averaged probability $p_{\text {dist }}$ of distinguishing the signals $\sigma_{\mathbf{i}}$ with equal prior probability $1 /\left(k !\left(\begin{array}{l}N \\ k\end{array}\right)\right)$ for arbitrary measurements $\Pi_{\mathbf{i}}$ is given by (see [4]):

$$
F=\frac{k !\left(\begin{array}{l}
N \\
k
\end{array}\right)}{d^{2 k}} p_{\text {dist }}, \quad p_{\text {dist }}=\frac{1}{k !\left(\begin{array}{l}
N \\
k
\end{array}\right)} \sum_{\mathbf{i} \in \mathcal{I}} \operatorname{Tr}\left(\Pi_{\mathbf{i}} \sigma_{\mathbf{i}}\right) .
$$

Now, our goal is to provide effectively computable lower bound on $p_{d i s t}$, which gives us the lower bound on $F$ of our protocol. Since we are interested in any feasible lower bound, for any $k \geq 1$, in (5) we take square-root measurements (SRM) of the form:

$$
\Pi_{\mathbf{i}}=\rho^{-1 / 2} \sigma_{\mathbf{i}} \rho^{-1 / 2} \quad \text { with } \quad \rho=\sum_{\mathbf{i} \in \mathcal{I}} \sigma_{i}
$$

The support of square-root measurements is always restricted to the support of the signals $\sigma_{\mathbf{i}}[14,30]$ making explicit calculations difficult. However, by formulating the generalisation of the statement of Lemma A.3 from [4] (see also Appendix B for details), we get a general lower bound on $p_{\text {dist }}$ :

$$
p_{d i s t} \geq \frac{1}{d^{N-k} \operatorname{Tr} \bar{\rho}^{2}}
$$

where $\bar{\rho}$ is a normalised version of the operator $\rho$ from (6). To use (7) one has to evaluate $\operatorname{Tr} \bar{\rho}^{2}$. The result (Appendix B) is the following:

Lemma 1. For the operator $\bar{\rho}=\rho / \operatorname{Tr} \rho$, we have

$$
\operatorname{Tr}\left(\bar{\rho}^{2}\right)=d^{-N-k}\left(\begin{array}{l}
N \\
k
\end{array}\right)^{-1}\left(\begin{array}{c}
d^{2}+N-1 \\
k
\end{array}\right) .
$$

Now we are in position to provide our lower bound for teleportation fidelity.

Theorem 2. The entanglement fidelity $F$ in MPBT deterministic scheme, with $N$ ports of dimension $d$ each, while teleporting $k \leq\lfloor N / 2\rfloor$ particles satisfies

$$
F \geq\left(\begin{array}{l}
N \\
k
\end{array}\right)\left(\begin{array}{c}
d^{2}+N-1 \\
k
\end{array}\right)^{-1} \geq\left(1-\frac{d^{2}-1}{d^{2}+N-k}\right)^{k} .
$$

For fixed $k$ the fidelity scales at least as $1-O(1 / N)$ and for $N \rightarrow \infty$ the fidelity goes to 1 .

The first bound in (9) follows from Lemma 1 and expression (5). The proof of second estimate, being technical, we delegate to Appendix B. The last sentence of the Theorem is obtained by applying the
Bernoulli inequality $(1-x)^{k} \geq 1-k x$ (valid for $x<1$ ) to the second bound. In particular, for qubits it reads:

$$
F \geq 1-\frac{3 k}{4+N-k}
$$

For $k=1$, the bound in (9) reduces to the bound for the standard non-optimal PBT [4], namely $F \geq$ $1-\frac{d^{2}-1}{d^{2}+N-1}$.

Now we compare performance of MPBT with the port based teleportation protocols by considering packaged PBT [28]. We shall compare MPBT protocols only with the mentioned versions of PBT, since the versions with large port dimension $d^{k}$ become ineffective very fast [9] and fidelity drops substantially. For our considerations we use both the original, i.e. non-optimal PBT protocol (Figure 1), where Alice and Bob share $N$ maximally entangled states, as well as the optimized version of PBT scheme (OPBT), where Alice optimises over shared state and measurements. Let us emphasize that in our scheme we do not perform any optimisation as it is done in OPBT case and, moreover, we compare with the lower bound in Theorem 2.

Let us denote by $F(N, k)$ the fidelity of teleportation, regardless of the scheme, of $k$ qubit systems through $N$ ports.

Packaged PBT In this version Alice to transmit $k$-system state to Bob has to divide all the ports in her possession in $N / k$ packages (see Figure 3 ). Then she runs independently $k$ separate PBT protocols with $N / k$ ports each. The total fidelity $F_{\text {pack }}(N, k)$ in the packaged version of PBT equals

$$
F_{\text {pack }}(N, k):=F(N / k, 1)^{k} .
$$

Please notice that optimal MPBT protocol is at least good as packaged PBT, since optimisation over the resource state includes all packaged schemes. Here however, we compare packaged versions of PBT with lower bound from Theorem 2, so it could happen in principle that the lower bound performs worse than packaged PBT.

In paper [28] it was shown that in case of qubits the quantity $F_{\text {pack }}(N, k)$ can be bounded from below as

$$
F_{p a c k}(N, k)=\left(1-\frac{3 k}{4 N}\right)^{k} \geq 1-\frac{3 k^{2}}{4 N} .
$$

However, it is easy to check that bound given through Theorem 2 outperforms bound (12) for $k \geq 4$. This motivates us to compare bound (9) with exact values of the packaged PBT given by (11). We know from [15], that the fidelity for qubit optimal PBT is given by $F=\cos ^{2}\left(\frac{\pi}{N+2}\right)$. Then in the packaged version entanglement fidelity reads

$$
F_{\text {pack }}^{O P B T}(N, k)=\cos ^{2 k}\left(\frac{\pi}{N / k+2}\right) .
$$




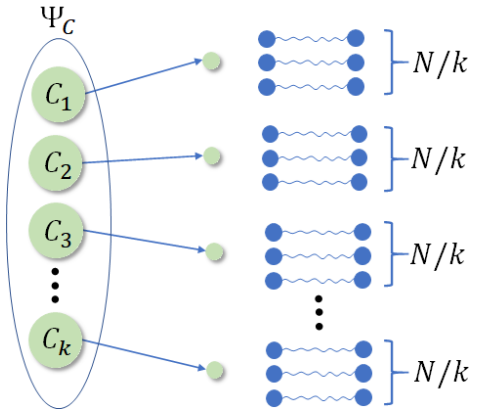

Figure 3: The packaged protocol for PBT. Alice to send a $k$-system state $\Psi_{C_{1} C_{2} \ldots C_{k}}$ to Bob divides all the ports into $N / k$ packages, and performs $k$ independent PBT protocols with $N / k$ ports each.

In Figure 4 we present comparison of the first bound from (9), plotted for various $k$ versus the fidelity of the packaged OPBT (13).

In our comparisons we use only the lower bound for non-optimal MPBT from Theorem 2, getting regions for which we perform better. For the exact values we obviously perform even better. In Appendix $\mathrm{C}$ we present an explicit expression for the entanglement fidelity in the qubit case, using angular momentum representation, and we compare it with the second bound from Theorem 2 .

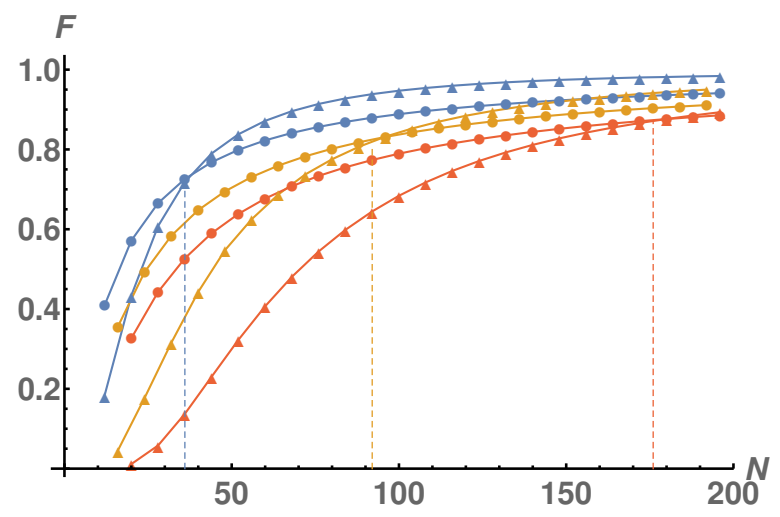

Figure 4: Comparison of the first bound from (9) from Theorem 2 (full circles, for $k=4$ marked with $\bullet, k=6 \bullet, k=8$ -) with exact values of the Pack. OPBT (13) (triangles, for fixed $k=4 \Delta, k=6 \Delta, k=8 \Delta$ ). For each fixed $k=4,6,8$ there exists $N$ for which the packaged version of OPBT outperforms the bound. We see, however, that for increasing number $k$ of teleported qubits, the region of supremacy of MPBT scheme increases quickly.

For fixed $k$ and small number of ports $N$ our first bound outperforms packaged OPBT from (11). However, for every fixed $k$ there is some point after which $F_{\text {pack }}^{O P B T}(N, k)$ dominates over the first (stronger) bound given by (9). Nevertheless, as $k$ increase this point shifts more rapidly. This suggests, that changing $k$ adaptively with growing $N$ shows the real advantage of the new protocol over the preexisting ones. We examine it in Section 4.1.

\begin{tabular}{c|c|c|c} 
& $F=0$ & $F_{\mathrm{cr}}$ & $F=1$ \\
\hline Pack.PBT & $\alpha>1 / 2$ & $\alpha_{\mathrm{cr}}=1 / 2, F_{\mathrm{cr}}=e^{-3 a^{2} / 4}$ & $\alpha<1 / 2$ \\
Pack.OPBT & $\alpha>2 / 3$ & $\alpha_{\mathrm{cr}}=2 / 3, F_{\mathrm{cr}}=e^{-\pi^{2} a^{3}}$ & $\alpha<2 / 3$ \\
$M P B T$ & - & $\alpha_{\mathrm{cr}}=1, \quad F_{\mathrm{cr}} \geq e^{-\frac{3 a}{1-a}}$ & $\alpha<1$ \\
\hline
\end{tabular}

Table 2: Comparison of the asymptotic behaviour of two variants of packaged PBT with MPBT in deterministic version, where $k=a N^{\alpha}$. By "cr" we denote the critical values of parameter $\alpha$ for which asymptotic value of $F$ exhibits a jump.

\section{Multiport versus standard PBT: large number of teleported qubits}

\subsection{Deterministic protocol}

We now compare performance of the MPBT protocol with the packaged $(\mathrm{O}) \mathrm{PBT}$ ones in the asymptotic regime, when we change the number of teleported particles adaptively. Namely, we shall assume that the number of teleported particles scales as $k(N)=a N^{\alpha}$.

For the case of MPBT we can formulate

Proposition 3. When $k / N \rightarrow 0$, the fidelity approaches 1. Moreover, for $k=\lfloor a N\rfloor$ where $a<1$ we have $\lim _{N \rightarrow \infty} F \geq \mathrm{e}^{-\left(d^{2}-1\right) \frac{a}{1-a}}$.

When $k=\lfloor a N\rfloor$ the statement of the Proposition follows directly from the calculation of the limit of the first bound given in (9). The case $k=o(N)$ follows from applying Bernoulli inequality $(1-x)^{k} \geq 1-k x$ (valid for $x<1$ ) to the second bound in (9).

The relation between $\alpha$ and the asymptotic fidelity of PBT and OPBT protocols is as follows. For the non-optimal $\mathrm{PBT}$ protocol the fidelity fulfils $F \approx$ $1-\frac{3}{4 N}=1-O(1 / N)[15]$. Thus, we can easily calculate $\lim _{N \rightarrow \infty} F_{\text {pack }}^{P B T}(N, k)$ for the non-optimal packaged PBT. It turns out that depending on whether $\alpha$ is greater, smaller or equal to particular value $\alpha_{c r}$ (which in this case equals $1 / 2$ ) the asymptotic fidelity equals 0,1 or a fixed value $F_{c r}$.

For $F_{\text {pack }}^{O P B T}(N, k)$ we can apply exactly the same reasoning, since in that case $F=\cos ^{2}\left(\frac{\pi}{N+2}\right) \approx 1-$ $\pi^{2} / N^{2}[15]$.

The results for all three schemes are presented in the Table 2 and the convergence for OPBT and MPBT protocols is depicted in the Figure 5.

MPBT scheme offers qualitative improvement over the original scheme for teleporting multipartite states. Even in optimal scheme of standard PBT (i.e. one, with optimal POVMs and the resource state) the number of teleported qubits can only scale as $N^{2 / 3}$, while in the original scheme, (where the resource state is just $N$ EPR pairs) the scaling is $N^{1 / 2}$.

\subsection{Probabilistic protocol}

Here we shall compare MPBT versus packaged PBT scheme in probabilistic scenario, where the figure of 


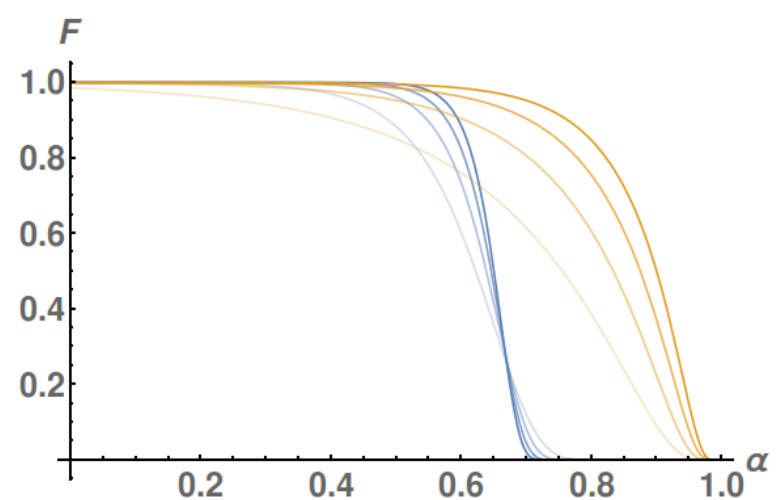

Figure 5: Asymptotic values of the lower bound on entanglement fidelity in MPBT (orange lines) compared to the fidelity of Pack. OPBT (blue lines), where $k=a N^{\alpha}, a=1, \alpha \in$ $(0,1)$, see Table 2. $N$ runs through $10^{2}, 10^{3}, 10^{4}$ and $10^{5}$ as the lines become thicker.

\begin{tabular}{c|c|l|c} 
& $p_{s}=0$ & \multicolumn{1}{|c|}{$p_{s, \mathrm{cr}}$} & $p_{s}=1$ \\
\hline Pack.PBT & $\alpha>1 / 3$ & $\alpha_{\mathrm{cr}}=1 / 3, p_{s, \mathrm{cr}}=e^{-c a^{3 / 2}}$ & $\alpha<1 / 3$ \\
Pack.OPBT & $\alpha>1 / 2$ & $\alpha_{\mathrm{cr}}=1 / 2, p_{s, \mathrm{cr}}=e^{-3 a^{2}}$ & $\alpha<1 / 2$ \\
$M P B T$ & $\alpha>1 / 2$ & $\alpha_{\mathrm{cr}}=1 / 2, \quad$ eq. $(15)$ & $\alpha<1 / 2$ \\
OMPBT & - & $\alpha_{\mathrm{cr}}=1, \quad p_{s, \mathrm{cr}}=(1+a)^{3}$ & $\alpha<1$ \\
\hline
\end{tabular}

Table 3: Table collects the comparison of the asymptotic behaviour of probability of success $p_{s}$ of all variants of packaged PBT with MPBT in probabilistic version when $k=a N^{\alpha}$. Here $c=\sqrt{8 / \pi}$. By "cr" we denote the critical values of parameter $\alpha$ for which asymptotic value of $p_{s}$ exhibits a jump.

merit is probability of success. Again we will assume that $k=a N^{\alpha}$. We do not have here such simple explicit bounds as those given in Theorem 2. We shall therefore use exact formula derived in companion paper by means of representation theoretic tools [30]. Basing on this result, in Appendix $\mathrm{C}$ we present exact formula for the probability of success in qubit case expressed in terms of angular momentum parameters which reads:

$$
p_{\text {succ }}(N, k)=\frac{1}{2^{N}} \frac{1}{N+1} \sum_{s=0\left(\frac{1}{2}\right)}^{\frac{N-k}{2}}(2 s+1)^{2}\left(\begin{array}{c}
N+1 \\
\frac{N-k}{2}-s
\end{array}\right) .
$$

Setting $k=a \sqrt{N}$ we can examine the dependence of (14) on $a$. We can observe, that when $a \rightarrow 0$ then $p_{\text {succ }} \rightarrow 1$. The latter is given (15), proven in Appendix $\mathrm{D}$ with the means of Central Limit type theorem, although in not completely straightforward way.

$\lim _{N \rightarrow \infty} p_{\text {succ }}= \begin{cases}2 \int_{0}^{\infty} x^{2} \frac{1}{\sqrt{2 \pi}} \mathrm{e}^{-\frac{(x+a)^{2}}{2}} d x, & k=a \sqrt{N}, a> \\ 1, & k=o(\sqrt{N})\end{cases}$

For PBT, in the case of original (i.e. nonoptimized) scheme [15] we have $p_{\text {succ }} \approx 1-c / \sqrt{N}$, where $c=\sqrt{\frac{8}{\pi}}$, and $p_{\text {succ }}=1-3 /(3+N) \approx 1-3 / N$ in the case of OPBT [15]. We may then apply the same reasoning

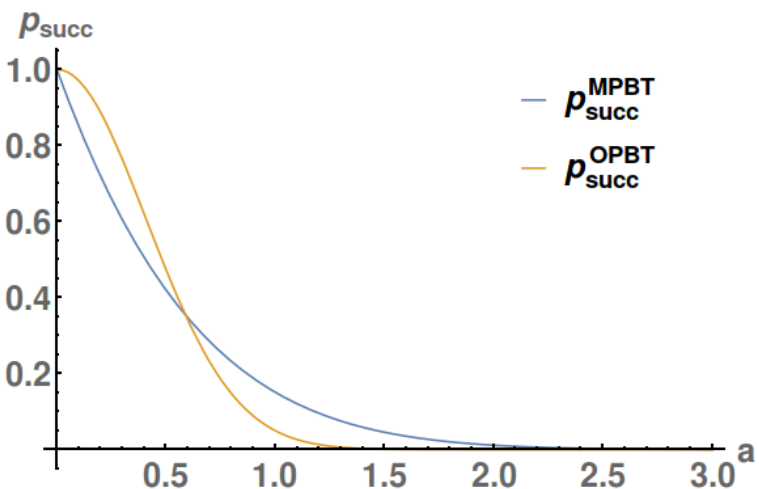

Figure 6: The limiting probability of success in the probabilistic schemes: Pack. OPBT(orange line) and MPBT (blue line) plotted as a function of $a$ when $k=a \sqrt{N}$, i.e. $\alpha=1 / 2$, see Table 3.

as in the deterministic case. Considering (14) we can see that

$$
\lim _{N \rightarrow \infty} p_{\text {succ }}^{M P B T}=1 \text { for } \alpha<1 / 2 .
$$

We can thus see that, unlike the deterministic protocol, the regions of asymptotic behaviour of $p_{\text {succ }}$ are separated by the same critical value $\alpha_{c r}=1 / 2$ for packaged OPBT and MPBT probablistic protocols. Plotting $p_{\text {succ }}^{M P B T}$ and $p_{\text {succ }}^{O P B T}$ as a function of $a$ in this borderline case, i.e. when $\alpha=\alpha_{c r}=1 / 2$ (see Figure 6 ), we observe the new protocol outperforms the previous one in the regime of large $a$.

However, one can refer to recent result concerning optimal MPBT [20], where remarkably simple expression for probability of success is evaluated:

$$
p_{\text {succ }}^{O M P B}=\prod_{m=2}^{d^{2}}\left(1-\frac{k}{N-1+m}\right) .
$$

This for $k=o(N)$ leads to $\lim _{N \rightarrow \infty} p_{\text {succ }}^{O M P B T}=1$. We have for $d=2$

$$
\lim _{N \rightarrow \infty} p_{\text {succ }}^{\text {OMPBT }}= \begin{cases}(1-a)^{3}, & k=a N \\ 1, & k=o(N)\end{cases}
$$

which outperforms the optimal PBT. This for $k=$ $o(N)$ leads to $\lim _{N \rightarrow \infty} p_{\text {succ }}^{O M P B T}=1$. Moreover, for $d=2$ and $k=a N$, that $\lim _{N \rightarrow \infty} p_{\text {succ }}^{O M P B T}=(1-a)^{3}$, which outperforms the optimal packaged PBT. We present the comparison of scaling of the packaged OPBT and OMPBT protocols in the Table 3 and Figure 7.

Finally, for a finite number of ports $N$ we have precise lower and upper bound for $p_{\text {succ }}$ in our protocol, which are presented in the Appendix D.

\section{Conclusions and Discussions}

We have addressed the problem of teleporting a large amount of quantum information. In particular 


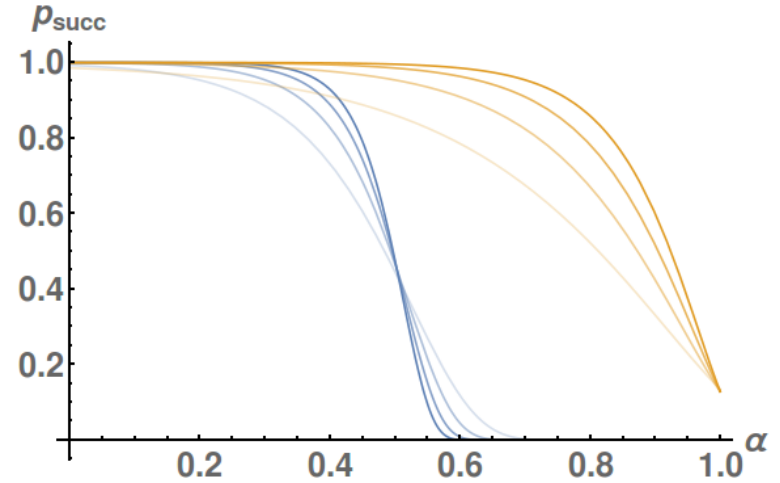

Figure 7: Probability of success, compared for Pack. OPBT (blue lines), and OMPBT (orange lines) protocols, where $k=a N^{\alpha}, a=\frac{1}{2}$, see Table 3. $N$ runs through $10^{2}, 10^{3}, 10^{4}$ and $10^{5}$ as the lines become thicker.

we analyse quantum multi-port teleportation protocol performing transmission of several quantum systems in one round. By examining improved lower bound on its performance, measured in the entanglement fidelity, we show that the protocol outperforms introduced earlier PBT protocols. The bound has been obtained by considering the teleportation process in our protocol as a state discrimination task and it depends only on global parameters like the number of ports, their dimension, and the number of teleported particles. In particular, we derived closed expressions for the entanglement fidelity and the probability of success in the described scheme for qubits in the picture of the angular momentum and using Gaussian approximation.

Further, we have shown that in general the number of systems to be teleported can be changed dynamically by the sender with the growing number of ports, still ensuring high efficiency in deterministic and probabilistic scheme. Even in the optimal PBT scheme, in deterministic case, fidelity can approach 1 for teleporting of up to $N^{2 / 3}$ particles, for our MPBT $N^{\alpha}$ particles can be teleported, if only $\alpha<1$. In probabilistic case, although the rates are equal and the teleportation achieving asymptotically unit probability of success is possible for number of teleported particles of the order $N^{\alpha}, \alpha<1 / 2$, both in MPBT and OPBT, we present result concerning optimal MPBT, which outperforms previous protocols [20].

We have thus showed that analysed protocol, while still requiring quite mild correction on Bob's site (just permuting his systems), exhibits qualitatively better "capacity" of transmission. Our results pave a novel way in teleportation and quantum communication in general. So far in quantum communication, while sending quantum information, one was interested in linear rate, i.e. the number of sent qubits per number of channel (or entangled pair) uses. On the other hand in quantum computing, it is very important to have teleportation with less correction on receiver's side. The port-based teleportation is protocol with virtually no correction. Here, we consider for the first time the issue of the amount of quantum information sent via such teleportation protocols. Our work implies, that within the realm of weakened correction the proper notion of communication efficiency is determined by asymptotic exponents in first place.

Still, there are a few open questions. First, except probabilistic OMPBT, we do not have exact asymptotic expressions for $d>2$. However, application of central limit theorem or adaptation techniques from [9] theorem should lead to the solution. In the case of deterministic OMPBT the problem of getting the asymptotic behaviour is more complex, since we do not have a closed formula for the fidelity. The fidelity there is given in terms of maximal eigenvalue of teleportation matrix [19, 30], for which we do not have an analytical formula yet, even for $k=1$. Finally, we leave the rigorous analysis of the recycling protocol introduced in [28] and its comparsion with our results for further project.

Acknowledgements MS, MM are supported through grant Sonatina 2, UMO2018/28/C/ST2/00004 from the Polish National Science Centre. Moreover, $\mathrm{MH}$ and MM thank the Foundation for Polish Science through IRAP project co-financed by the EU within the Smart Growth Operational Programme (contract no. 2018/MAB/5). $\mathrm{MH}$ also acknowledges support from the National Science Centre, Poland, through grant OPUS 9, 2015/17/B/ST2/01945. MM and PK would like to thank ICTQT Centre (University of Gdańsk) for hospitality where part of this work has been done.

\section{A Symmetries in Multi-port teleportation scheme}

Before we present argumentation leading us to the proof of Lemma 1 from the main text, we introduce here concepts of permutation operator and its partial transposition. Most of the formalism presented here can be also find in $[17,30]$. Let us consider a representation $V$ of the permutation group $S(n)$ in the space $\mathcal{H} \equiv\left(\mathbb{C}^{d}\right)^{\otimes n}$, defined in the following way

$$
\forall \pi \in S(n) \quad V(\pi) .\left|e_{i_{1}}\right\rangle \otimes\left|e_{i_{2}}\right\rangle \otimes \cdots \otimes\left|e_{i_{n}}\right\rangle:=\left|e_{i_{\pi^{-1}(1)}}\right\rangle \otimes\left|e_{i_{\pi^{-1}(2)}}\right\rangle \otimes \cdots \otimes\left|e_{i_{\pi^{-1}(n)}}\right\rangle
$$

where the set $\left\{\left|e_{i}\right\rangle\right\}_{i=1}^{d}$ is an orthonormal basis of the space $\mathbb{C}^{d}$, and $d$ stands for the dimension. We drop here the lower index in every $i$, since it labels only position of the basis in tensor product $\left(\mathbb{C}^{d}\right)^{\otimes n}$. This representation 
is in fact a unitary matrix representation, since it is given with respect to prescribed basis $\left\{\left|e_{i}\right\rangle\right\}_{i=1}^{d}$ in the space $\mathbb{C}^{d}$, and for every $\pi \in S(n)$ the corresponding matrix representation $V(\pi)$ is called a permutation operator. The representation $V$ of $S(n)$ extends in a natural way to the representation of the group algebra $\mathbb{C}[S(n)]$ and in this way we get the algebra of permutation operators

$$
\mathcal{A}_{n}(d) \equiv \operatorname{span}_{\mathbb{C}}\{V(\pi): \pi \in S(n)\} .
$$

Having the above definition of the algebra $\mathcal{A}_{n}(d)$, we can introduce a new complex algebra - algebra of the partially transposed permutation operators:

$$
\mathcal{A}_{n}^{(k)}(d) \equiv \operatorname{span}_{\mathbb{C}}\left\{V^{(k)}(\pi): \pi \in S(n)\right\}
$$

where the symbol $(k)$ denotes partial transpose operation with respect to last $k$ systems in the space $\left(\mathbb{C}^{d}\right)^{\otimes n}$. Now, we show that the operator $\rho$ defined in expression (6) from the main text is an element of the algebra $\mathcal{A}_{n}^{(k)}(d)$. Let us denote by $n=N+k$ the number of all systems involved in the teleportation process on Alice's (Bob's) side and introduce the following mapping of indices

$$
\forall \mathbf{i} \in \mathcal{I} \quad B_{\mathbf{i}} \mapsto B=\underbrace{B_{n-k+1} B_{n-k+2} \cdots B_{n}}_{k} .
$$

Having that we can work with the equivalent form of the operator $\rho$, which is given through the following expression

$$
\rho=\sum_{\mathbf{i} \in \mathcal{I}} \sigma_{i}=\frac{1}{d^{N-k}} \sum_{\mathbf{i} \in \mathcal{I}} \mathbf{1}_{\bar{A}_{\mathbf{i}}} \otimes P_{A_{\mathbf{i}} B}^{+},
$$

where for a given index $\mathbf{i}=\left\{i_{1}, i_{2}, \ldots, i_{k}\right\}$ the operator $\mathbf{1}_{\bar{A}_{\mathbf{i}}} \otimes P_{A_{\mathbf{i}} B}^{+}$is of the following form

$$
\begin{aligned}
\sigma_{\mathbf{i}} & =\mathbf{1}_{\bar{A}_{\mathbf{i}}} \otimes P_{A_{\mathbf{i}} B}^{+}=\mathbf{1}_{\bar{A}_{\mathbf{i}}} \otimes P_{i_{1}, n-k+1}^{+} \otimes P_{i_{2}, n-k+2}^{+} \otimes \cdots \otimes P_{i_{k}, n}^{+} \\
& =\frac{1}{d^{N}} \mathbf{1}_{\bar{A}_{\mathbf{i}}} \otimes V^{t_{n-k+1}}\left[\left(i_{1}, n-k+1\right)\right] \otimes V^{t_{n-k+2}}\left[\left(i_{2}, n-k+2\right)\right] \otimes \cdots \otimes V^{t_{n}}\left[\left(i_{k}, n\right)\right] .
\end{aligned}
$$

By $t_{n-k+1}, t_{n-k+2}, \ldots, t_{n}$ we denote transposition with respect to systems on positions $n-k+1, n-k+2, \ldots, n$ respectively. Next, we have to rewrite $\rho$ in more convenient form for our further considerations. First, we observe that we can distinguish an index $\mathbf{i}_{0}=\{n-k, n-k-1, \ldots, n-2 k+1\}$, for which the corresponding signal is called canonical:

$$
\begin{aligned}
\sigma_{\mathbf{i}_{0}} & =\frac{1}{d^{N}} \mathbf{1}_{\bar{A}_{\mathbf{i}_{0}}} \otimes V^{t_{n-k+1}}[(n-k, n-k+1)] \otimes V^{t_{n-k+2}}[(n-k-1, n-k+2)] \otimes \cdots \otimes V^{t_{n}}[(n-2 k+1, n)] \\
& =\frac{1}{d^{N}}\left(\mathbf{1}_{\bar{A}_{\mathbf{i}_{0}}} \otimes V[(n-k, n-k+1)] \otimes V[(n-k-1, n-k+2)] \otimes \cdots \otimes V[(n-2 k+1, n)]\right)^{t_{n-k+1} t_{n-k+2} \cdots t_{n}} \\
& =\frac{1}{d^{N}} V^{(k)}
\end{aligned}
$$

by $V$ we denote the total permutation operator in the bracket with this specific composition of permutations, including the identity operator. Having the definition of $\sigma_{\mathbf{i}_{0}}$, let us observe that any other signal can be obtained from it by acting of $V(\tau)$, where $\tau$ is permutation from the coset $\mathcal{S}_{n, k}=S(n-k) / S(n-2 k)$, so

$$
\rho=\frac{1}{d^{N}} \sum_{\tau \in \mathcal{S}_{n, k}} V(\tau) V^{(k)} V^{\dagger}(\tau) .
$$

This clearly shows that the operator $\rho$, as well as every operator $\sigma_{\mathbf{i}}$ belongs to the algebra $\mathcal{A}_{n}^{(k)}(d)$ defined in (21). The cardinality of $\mathcal{S}_{n, k}$ gives us number of all possible signals $k !\left(\begin{array}{l}N \\ k\end{array}\right)$. Since all the operators $V(\tau)$ are invariant with respect to the composition of partial transpositions $(k)$, we can rewrite $\rho$ from $(26)$ as in the following definition:

Definition 4. For $N$ ports the operator $\rho$ from expression (26) can be re-written in the following form:

$$
\rho \equiv \sum_{a_{1}, a_{2}, \ldots, a_{k}=1}^{n-k} V^{(k)}\left[\left(a_{k}, n-k+1\right)\left(a_{k-1}, n-k+2\right) . \cdots\left(a_{1}, n\right)\right]
$$

where all numbers $a_{1}, a_{2}, \ldots, a_{k}$ are different and $k \leq\left\lfloor\frac{N}{2}\right\rfloor$. For compactness of the further calculations we drop here the normalisation constant $1 / d^{N}$ in form of every signal $\sigma_{\mathbf{i}}$. 


\section{B Proof of Lemma 1 and Theorem 1 from the main text}

Having discussion on symmetries in multi-port based teleportation protocols we are in position to compute the lower bound on the entanglement fidelity $F$. As it was pointed in the main text, and in [4, 14], the entanglement fidelity $F$ can be connected with probability of success of the state discrimination $p_{\text {dist }}$ of the ensemble $\mathcal{E}$ :

$$
\mathcal{E}=\left\{\frac{1}{k !\left(\begin{array}{c}
N \\
k
\end{array}\right)}, \sigma_{\mathbf{i}}\right\}
$$

where $\sigma_{\mathbf{i}}$ are the signal states described in Section A. It can be shown that $F$ and $p_{\text {dist }}$ are related to each other by the following relation

$$
F=\frac{k !\left(\begin{array}{c}
N \\
k
\end{array}\right)}{d^{2 k}} p_{\text {dist }}, \quad p_{\text {dist }}=\frac{1}{k !\left(\begin{array}{l}
N \\
k
\end{array}\right)} \sum_{\mathbf{i} \in \mathcal{I}} \operatorname{Tr}\left(\Pi_{\mathbf{i}} \sigma_{\mathbf{i}}\right) \geq \frac{1}{k !\left(\begin{array}{l}
N \\
k
\end{array}\right) r \operatorname{Tr} \bar{\rho}^{2}},
$$

where $r=\left(1 /\left(k !\left(\begin{array}{c}N \\ k\end{array}\right)\right)\right) \sum_{\mathbf{i} \in \mathcal{I}} \operatorname{rank}\left(\sigma_{\mathbf{i}}\right)=d^{N-k}$, and the lower bound on $p_{\text {dist }}$ is a simple generalisation of Lemma A.3 from [4]. This means that $F$ is bounded from the below as

$$
F \geq \frac{1}{d^{N+k} \operatorname{Tr}\left(\bar{\rho}^{2}\right)}
$$

where the operator $\bar{\rho}$ is normalised version $(\operatorname{Tr} \bar{\rho}=1)$ of the operator $\rho$ from Definition 4 :

$$
\bar{\rho}=\frac{\rho}{\operatorname{Tr}(\rho)}=\frac{1}{d^{N} k !\left(\begin{array}{l}
N \\
k
\end{array}\right)} \rho,
$$

since we can observe that for operator $\rho$ from Definition 4 the following holds

$$
\begin{aligned}
\operatorname{Tr}(\rho) & =d^{n-k}(n-k)(n-k-1) \cdot \ldots \cdot(n-2 k+1)=d^{N} N(N-1) \cdot \ldots \cdot(N-k+1)=d^{N} k !\left(\begin{array}{c}
N \\
k
\end{array}\right) \\
& =d^{N} \frac{N !}{(N-k) !}
\end{aligned}
$$

where $N=n-k$ denotes number of ports. It means that normalised operator $\bar{\rho}$ can be written finally as

$$
\bar{\rho}=\frac{\rho}{\operatorname{Tr}(\rho)}=\frac{1}{d^{N} \frac{N !}{(N-k) !}} \rho .
$$

Later on we use form of $\bar{\rho}$ from expression (33).

The above considerations show that to compute the right-hand side of (30) the crucial is evaluation of $\operatorname{Tr}\left(\rho^{2}\right)$. The calculation of $\operatorname{Tr}\left(\rho^{2}\right)$ is technically complicated and lengthy and it contains a few intermediate steps and auxiliary results which we present separately as Lemma 6 to 10 . The final result, i.e. the formula for $\operatorname{Tr}\left(\rho^{2}\right)$ is given in Theorem 11 . We will use in the derivation of this result the following useful notation

Notation 5. We define inductively a sequence of transpositions

$$
\begin{aligned}
& L_{1}=\left(a_{1}, b_{1}\right), L_{2}=\left(a_{2}, L_{1}\left(b_{2}\right)\right), L_{3}=\left(a_{3}, L_{2} L_{1}\left(b_{3}\right)\right), \ldots, L_{k}=\left(a_{k}, L_{k-1} \cdots L_{2} L_{1}\left(b_{k}\right)\right), \\
& L_{1}^{\prime}=\left(b_{1}, a_{1}\right), L_{2}^{\prime}=\left(b_{2}, L_{1}^{\prime}\left(a_{2}\right)\right), L_{3}^{\prime}=\left(b_{3}, L_{2}^{\prime} L_{1}^{\prime}\left(a_{3}\right)\right), \ldots, L_{k}^{\prime}=\left(b_{k}, L_{k-1}^{\prime} \cdots L_{2}^{\prime} L_{1}^{\prime}\left(a_{k}\right)\right),
\end{aligned}
$$

where $(a, b)$ is simply a transposition acting on some $c$ as

$$
(a, b)(c)= \begin{cases}c, & \text { when } a, b \neq c \\ b, & \text { when } a=c \\ a, & \text { when } b=c\end{cases}
$$

and where the numbers $a_{1}, a_{2}, \ldots, a_{k}$ are different and similarly the numbers $b_{1}, b_{2}, \ldots, b_{k}$ are different. For such a transpositions we define reversed transpositions also defined on the same numbers $a_{1}, a_{2}, \ldots, a_{k}$ and $b_{1}, b_{2}, \ldots, b_{k}$ in the following way so in the primed transpositions the numbers $a_{1}, a_{2}, \ldots, a_{k}$ and $b_{1}, b_{2}, \ldots, b_{k}$ are interchanged. 
The above transpositions satisfy the relation which easily follows from the structure of the transpositions

Lemma 6. We have

$$
L_{s}^{\prime} L_{s-1}^{\prime} \cdots L_{1}^{\prime}=L_{1} L_{2} \cdots L_{s}, \quad s=1, \ldots, k .
$$

Using introduced notation we may derive the following composition rule

Lemma 7. Let the numbers $1 \leq a_{1}, a_{2}, \ldots, a_{k} \leq n-k$ are different and similarly numbers $1 \leq b_{1}, b_{2}, \ldots, b_{k} \leq$ $n-k$ are different and $k \leq\left\lfloor\frac{n}{2}\right\rfloor$, then

$$
\begin{aligned}
& V^{(k)}\left[\left(a_{k}, n-k+1\right)\left(a_{k-1}, n-k+2\right) \cdots\left(a_{1}, n\right)\right] V^{(k)}\left[\left(b_{k}, n-k+1\right)\left(b_{k-1}, n-k+2\right) \cdots\left(b_{1}, n\right)\right]
\end{aligned}
$$

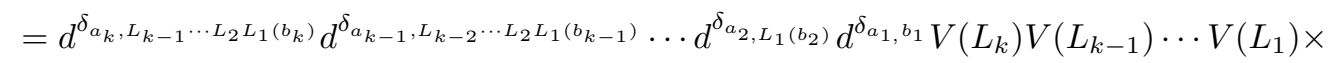

$$
\begin{aligned}
& \times V^{(k)}\left[\left(b_{k}, n-k+1\right)\left(b_{k-1}, n-k+2\right) \cdots\left(b_{1}, n\right)\right] .
\end{aligned}
$$

Applying the statement of Proposition 7 twice, together with Lemma 6, we get

Lemma 8. Let the numbers $1 \leq a_{1}, a_{2}, \ldots, a_{k} \leq n-k$ are different and similarly the numbers $1 \leq b_{1}, b_{2}, \ldots, b_{k} \leq$ $n-k$ are different and $k \leq\left\lfloor\frac{n}{2}\right\rfloor$, then

$$
\begin{aligned}
& V^{(k)}\left[\left(a_{k}, n-k+1\right)\left(a_{k-1}, n-k+2\right) \cdots\left(a_{1}, n\right)\right] V^{(k)}\left[\left(b_{k}, n-k+1\right)\left(b_{k-1}, n-k+2\right) \cdots\left(b_{1}, n\right)\right] \times \\
& \times V^{(k)}\left[\left(a_{k}, n-k+1\right)\left(a_{k-1}, n-k+2\right) \cdots\left(a_{1}, n\right)\right]
\end{aligned}
$$

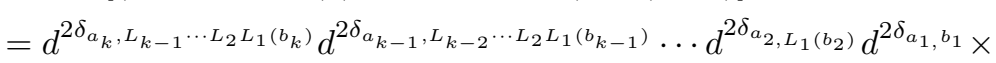

$$
\begin{aligned}
& \times V^{(k)}\left[\left(a_{k}, n-k+1\right)\left(a_{k-1}, n-k+2\right) \cdots\left(a_{1}, n\right)\right] .
\end{aligned}
$$

From expression (39) we get immediately

Corollary 9. Let the numbers $1 \leq a_{1}, a_{2}, \ldots, a_{k} \leq n-k$ are different and similarly numbers $1 \leq b_{1}, b_{2}, \ldots, b_{k} \leq$ $n-k$ are different and $k \leq\left\lfloor\frac{n}{2}\right\rfloor$, then

$$
\begin{aligned}
& \operatorname{Tr}\left[V^{(k)}\left[\left(a_{k}, n-k+1\right)\left(a_{k-1}, n-k+2\right) \cdots\left(a_{1}, n\right)\right] V^{(k)}\left[\left(b_{k}, n-k+1\right)\left(b_{k-1}, n-k+2\right) \cdots\left(b_{1}, n\right)\right]\right]
\end{aligned}
$$

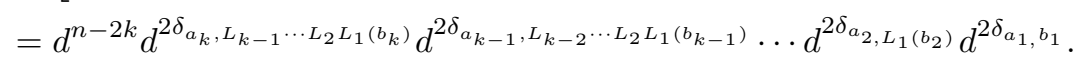

Using equation (40) we get that

$\operatorname{Tr}\left(\rho^{2}\right)=\sum_{a_{i}} \sum_{b_{i}} \operatorname{Tr}\left[V^{(k)}\left[\left(a_{k}, n-k+1\right)\left(a_{k-1}, n-k+2\right) \cdots\left(a_{1}, n\right)\right] V^{(k)}\left[\left(b_{k}, n-k+1\right)\left(b_{k-1}, n-k+2\right) \cdots\left(b_{1}, n\right)\right]\right.$,

where the summation is all over pairwise different $1 \leq a_{1}, a_{2}, \ldots, a_{k} \leq n-k$ and $1 \leq b_{1}, b_{2}, \ldots, b_{k} \leq n-k$, is equal to

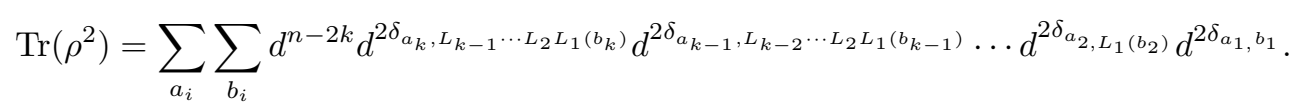

In order to calculate this sum we need the following

Lemma 10. Using Notation 5 we have the following property:

$$
b_{s} \neq b_{1}, b_{2}, \ldots, b_{s-1} \Rightarrow L_{s-1} L_{s-2} \cdots L_{1}\left(b_{s}\right) \neq a_{1}, a_{2}, \ldots, a_{k-1}, \quad s=1, \ldots, k .
$$

In the particular case, for $k=2$, the statement of the above lemma reduces to

$$
b_{2} \neq b_{1} \Rightarrow L_{1}\left(b_{2}\right) \neq a_{1} .
$$

Applying Lemma 10 to each sum appearing on the $R H S$ of equation for $\operatorname{Tr}\left(\rho^{2}\right)$ we are in position to formulate the main result of this section:

Theorem 11. Let $\rho$ be as in Definition 4, then

$$
\operatorname{Tr}\left(\rho^{2}\right)=d^{n-2 k}(n-2 k-1)(n-2 k-2) \cdots(n-k)\left(d^{2}+n-2 k\right)\left(d^{2}+n-2 k+1\right) \cdots\left(d^{2}+n-k-1\right),
$$

or equivalently, in a more compact form

$$
\operatorname{Tr}\left(\rho^{2}\right)=d^{N-k} \frac{N !}{(N-k) !} \frac{\left(d^{2}+N-1\right) !}{\left(d^{2}+N-k-1\right) !}
$$

where $N=n-k$ is number of port in MPBT protocol. 
Proof. In order to better explain the role of Lemma 10 in the proof, let us consider first the case of $k=2$, where we have

$$
\operatorname{Tr}\left(\rho^{2}\right)=d^{n-4} \sum_{b_{i}}\left(\sum_{a_{i}} d^{2 \delta_{a_{2}, \tau}\left(a_{1}, b_{1}\right)^{\left(b_{2}\right)}} d^{2 \delta_{a_{1}, b_{1}}}\right)=d^{n-4} \sum_{b_{i}}\left(\sum_{a_{1}} \sum_{a_{2} \neq a_{1}} d^{2 \delta_{a_{2}, \tau}\left(a_{1}, b_{1}\right){ }^{\left(b_{2}\right)}} d^{2 \delta_{a_{1}, b_{1}}}\right),
$$

where $1 \leq a_{1}, a_{2} \leq n-2$ and $1 \leq b_{1}, b_{2}, \leq n-2$ are pairwise different. Now using Lemma 10 we get

$$
\sum_{a_{2} \neq a_{1}} d^{2 \delta_{\left.a_{2}, \tau_{\left(a_{1}, b_{1}\right)}\right)^{\left(b_{2}\right)}}}=d^{2}+n-4
$$

since $a_{2}$ runs over the set $\{1,2, \ldots, n-2\} \backslash\left\{a_{1}\right\}$ exactly once is equal to the number $\tau_{\left(a_{1}, b_{1}\right)}\left(b_{2}\right) \neq a_{1}$ and $n-4$ times is not equal to $\tau_{\left(a_{1}, b_{1}\right)}\left(b_{2}\right)$. This implies

$$
\begin{aligned}
\operatorname{Tr}\left(\rho^{2}\right) & =d^{n-4}\left(d^{2}+n-4\right) \sum_{b_{1}} \sum_{b_{2} \neq b_{1}}\left(\sum_{a_{1}} d^{2 \delta_{a_{1}, b_{1}}}\right)=d^{n-4}(n-3)\left(d^{2}+n-4\right) \sum_{b_{1}=1}^{n-2} \sum_{a_{1}=1}^{n-2} d^{2 \delta_{a_{1}, b_{1}}} \\
& =d^{n-4}(n-2)(n-3)\left(d^{2}+n-3\right)\left(d^{2}+n-4\right) .
\end{aligned}
$$

For general case the way of proving is the same but we have more steps.

We illustrate the statement of Lemma 11 by two following examples:

EXAMPLE 12 Let $k=1$, then

$$
\operatorname{Tr}\left(\rho^{2}\right)=d^{n-2}(n-1)\left(d^{2}+n-2\right)
$$

This expression can be also evaluated using group-theoretic approach, see the proof of Theorem 52 on page 28 in [18].

EXAMPLE 13 Let $k=2$, then

$$
\operatorname{Tr}\left(\rho^{2}\right)=d^{n-4}(n-2)(n-3)\left(d^{2}+n-3\right)\left(d^{2}+n-4\right) .
$$

Here, the group-theoretic approach would demand new summation rules for the irreducible representations, which are yet not know.

All the above described considerations are in fact proof of Lemma 1 from the main text. Indeed, taking expression (46) and (32) we write the following for the normalised operator $\bar{\rho}$ from (33):

$$
\begin{aligned}
\operatorname{Tr}\left(\bar{\rho}^{2}\right) & =\frac{\operatorname{Tr}\left(\rho^{2}\right)}{(\operatorname{Tr}(\rho))^{2}}=\frac{d^{N-k}}{d^{2 N}} \frac{\frac{N !}{(N-k) !} \frac{\left(d^{2}+N-1\right) !}{\left(d^{2}+N-k-1\right) !}}{\left(\frac{N !}{(N-k) !}\right)^{2}} \\
& =d^{-N-k}\left[\frac{N !}{(N-k) !}\right]^{-1} \frac{\left(d^{2}+N-1\right) !}{\left(d^{2}+N-k-1\right) !} \\
& =d^{-N-k}\left(\begin{array}{l}
N \\
k
\end{array}\right)^{-1}\left(\begin{array}{c}
d^{2}+N-1 \\
k
\end{array}\right) .
\end{aligned}
$$

Now, using relation $n=N+k$ and expression (32) we rewrite (30) as

$$
F \geq \frac{1}{d^{N+k} \operatorname{Tr}\left(\bar{\rho}^{2}\right)}=\left(\begin{array}{c}
N \\
k
\end{array}\right)\left(\begin{array}{c}
d^{2}+N-1 \\
k
\end{array}\right)^{-1}
$$

which recovers the first bound (9) from Theorem 2 in the main text. To prove the second bound in (9) from the same theorem let us observe the following:

$$
\left(\begin{array}{l}
N \\
k
\end{array}\right)\left(\begin{array}{c}
d^{2}+N-1 \\
k
\end{array}\right)^{-1}=\prod_{s=0}^{k-1}\left(1-\frac{d^{2}-1}{d^{2}+N-1-s}\right) \geq\left(1-\frac{d^{2}-1}{d^{2}+N-k}\right)^{k}
$$

Now, let us prove that the entanglement fidelity $F$ scales at least as $1-O(1 / N)$ and goes to 1 with $N \rightarrow \infty$. To see this is is enough to apply the Bernoulli inequality $(1-x)^{k} \geq 1-k x$ (valid for $\left.x<1\right)$ to $\left(1-\frac{d^{2}-1}{d^{2}-N-k}\right)^{k}$. 
There is also an alternative proof of the fidelity scaling, showing interesting connection with the symmetric polynomials. To show it, first we have to derive a different lower bound for the entanglement fidelity exploiting symmetric polynomials. Defining variables $x_{s} \equiv 1 /\left(d^{2}+N-s-1\right)$ for $s=0, \ldots, k-1$, and completely symmetric polynomials $S_{l}\left(x_{1}, x_{2}, \ldots, x_{k}\right)$ in variables $x_{1}, x_{2}, \ldots, x_{k}$, for $l \in \mathbb{N}$. For example we have:

$$
S_{0}\left(x_{1}, x_{2}, \ldots, x_{k}\right)=1, \quad S_{1}\left(x_{1}, x_{2}, \ldots, x_{k}\right)=x_{1}+x_{2}+\ldots+x_{k}, \quad S_{2}\left(x_{1}, x_{2}, \ldots, x_{k}\right)=\sum_{1 \leq j \leq l \leq k} x_{j} x_{l} \text { etc. }
$$

Using the above, we can write the bound (53) with (54) as

$$
F \geq \prod_{s=0}^{k-1}\left(1-\frac{d^{2}-1}{d^{2}+N-s-1}\right)=\sum_{l=0}^{k}(-1)^{l}\left(d^{2}-1\right)^{l} S_{l}\left(x_{1}, x_{2}, \ldots, x_{k}\right) .
$$

To evaluate the leading term in obtained bounds on the entanglement fidelity we have to use observation that we can write the right-hand side of (9) in terms of symmetric polynomials $S_{l}\left(x_{1}, \ldots, x_{k}\right)$ with $x_{s}=1 /\left(d^{2}+N-s-1\right)$ for $s=0, \ldots, k-1$ :

$$
F \geq \sum_{l=0}^{k}(-1)^{l}\left(d^{2}-1\right)^{l} S_{l}\left(x_{1}, x_{2}, \ldots, x_{k}\right)=1-\sum_{s=0}^{k-1} \frac{d^{2}-1}{d^{2}+N-s-1}+O\left(1 / N^{2}\right) .
$$

We then see from (57) that when $k$ is fixed and $N$ goes to infinity, the right hand side approaches 1.

In the end of this appendix we briefly explain the flow of the reasoning presented [28]. The idea presented there is also based on the state discrimination problem and computing $\operatorname{Tr} \bar{\rho}^{2}$. However, argument for computing trace from the composition $\sigma_{\mathbf{i}} \sigma_{\mathbf{j}}$, for maximally entangled pairs fully overlapping, does not take into account that the resulting operators are in general permutation operators with different number of disjoint cycles. The final trace depends on the number of disjoint cycles and cannot be characterised only by global parameters, but has to take into account the interior structure of permutations. We illustrate this in the example below.

EXAMPLE 14 Let us consider two signals for $n=6, k=2$, and arbitrary $d$ of the form

$$
\sigma_{1}=\frac{1}{d^{4}} V^{(2)}[(45)(36)], \quad \sigma_{2}=\frac{1}{d^{4}} V^{(2)}[(35)(46)]
$$

Trace from their overlap, according to Corollary (9), equals to

$$
\operatorname{Tr}\left(\sigma_{1} \sigma_{2}\right)=\frac{1}{d^{8}} \operatorname{Tr}\left(V^{(2)}[(45)(36)] V^{(2)}[(35)(46)]\right)=\frac{d^{2}}{d^{8}} d^{2 \delta_{4, L_{1}(3)}} d^{\delta_{3,4}}=\frac{d^{2}}{d^{8}} d^{2 \delta_{4,4}}=\frac{1}{d^{4}},
$$

since $L_{1}(3)=(3,4)[3]=4$ (see Notation 5). However, in [28] authors argue that the value of $\operatorname{Tr}\left(\sigma_{1} \sigma_{2}\right)$ equals to $1 / d^{6}$, or in general $1 / d^{N+k}$, which is in contradiction with mentioned Corollary 9. Note that this particular example can be evaluated directly, without referring to the corollary, by exploiting, in particular, the simple fact, that $\operatorname{Tr} A^{\Gamma} B^{\Gamma}=\operatorname{Tr} A B$ for operators $A, B$, where $\Gamma$ is partial transpose, and the fact that trace of operator of permutation is equal to $d^{c}$ where $c$ is number of cycles of the permutation, including trivial cycles.

Fortunately, some part of argumentation from [28] can be saved. Namely, quantity $1 / d^{N+k}$ always lower bounds $\operatorname{Tr}\left(\sigma_{\mathbf{i}} \sigma_{\mathbf{j}}\right)$ for signals with overlapping maximally entangled states. This means that the result in [28] actually gives lower bound on $\operatorname{Tr} \bar{\rho}^{2}$ and then on entanglement fidelity. Here, we do not compute bound on $\operatorname{Tr} \bar{\rho}^{2}$, but we evaluate it explicitly. This leads us to more effective and elegant lower bound on efficiency of the deterministic MPBT.

\section{Derivation of the entanglement fidelity and probability of success for qubits}

Before we present here the main consideration leading us to closed expressions for the entanglement fidelity and average probability of success, we introduce basic ideas concerning representation theory of permutation group $S(n)$. For more details we refer reader to $[12,13]$

Every partition (shape) of natural number $n$ is a sequence $\alpha=\left(\alpha_{1}, \ldots, \alpha_{r}\right)$ satisfying

$$
\forall_{i} \alpha_{i} \geq 0, \quad \alpha_{1} \geq \alpha_{2} \geq \ldots \geq \alpha_{r}, \quad \sum_{i=i}^{r} \alpha_{i}=n
$$

where $r \in\{1, \ldots, n\}$. Every such partition corresponds with some frame, which is called Young frame. The Young frame associated with partition $\mu$ is the array formed by $n$ boxes with $l$ left-justified rows. 
EXAmPle 15 Let us take $n=4$. Allowed partitions are $\alpha_{1}=(4), \alpha_{2}=(3,1), \alpha_{3}=(2,2), \alpha_{4}=(2,1,1), \alpha_{5}=$ $(1,1,1,1)$ and they correspond to the following Young frames:

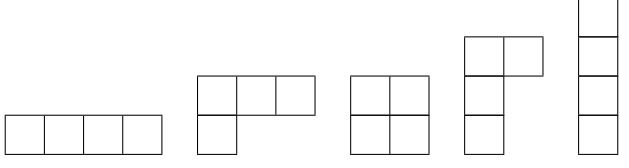

Any irreducible representation (irrep) of symmetric group $S(n)$ is labelled by Young frames with $n$ boxes. From above example we see that for $n=4$ we have five possible irreps - because we have only five different Young frames.

A Standard Young Tableaux (SYT) is a Young frame of shape $\alpha$ of $n$ objects, such that labels occur increasing in every row from left to the right, and increasing in every column from top to the downwards. One can see that every number between 1 and $n$ occurs only once.

EXAmple 16 For the case $n=4$ and $\alpha=(2,1,1)$ we have three possible fillings

\begin{tabular}{|c|c|c|}
\hline \begin{tabular}{l|l}
1 & 2
\end{tabular} & \begin{tabular}{|l|l|l|}
1 & 3 \\
\end{tabular} & \\
\hline 3 & 2 & 2 \\
\hline 4 & \begin{tabular}{|l|}
4 \\
\end{tabular} & 3 \\
\hline
\end{tabular}

The number of STYs for given shape $\alpha$ and natural number $n$ can be obtained by combinatorial rules. It turns out that the dimension $d_{\alpha}$ of an irreducible representation of symmetric group $S(n)$ for fixed $\alpha$ is equal to the number of SYTs.

A semi-standard Young Tableaux (SSYT) is a Young frame of shape $\alpha$ of $n$ boxes filled with numbers $\{1,2, \ldots, s\}$ for some $s$, such that rows are weakly and columns are strictly increasing.

EXAMPLE 17 For the case $n=4, \alpha=(3,1)$, and set $\{1,2\}$, we have three possible fillings

\begin{tabular}{|l|l|l|l|l|l|l|l|l|}
\hline 1 & 1 & 1 \\
\hline 2 & & 1 & 2
\end{tabular}$\quad$\begin{tabular}{|l|l|l|}
\hline 1 & 2 & 2 \\
\hline 2 & \\
\hline
\end{tabular}

The total number of SSYTs also can be computed by combinatorial rules, and this number equals to the multiplicity $m_{\alpha}$ in the space $\left(\mathbb{C}^{d}\right)^{\otimes n}$ by plugging $s=d$. One can notice, that whenever $d$ is smaller than the length of the longest column in $\alpha$ the corresponding multiplicity equals zero. This means that such irrep does not occur into decomposition. For example considering the qubit case, when $d=2$, only three first Young frames from (61) appear. More formally, considering representations of $S(n)$ on space $\left(\mathbb{C}^{d}\right)^{\otimes n}$, where $d$ denotes dimension, the irreducible representations of $S(n)$ are labelled by Young frames with $n$ boxes, restricted to the ones with at most $d$ rows. We denote this fact by $\alpha \vdash_{d} n$ (in short $\alpha \vdash n$ when it is clear from the context).

Additionally, the symbol $\mu \in \alpha$ denotes a Young frames $\mu \vdash n$ obtained from a Young frame $\alpha \vdash n-k$ by adding $k$ boxes one by one, getting in every step a valid Young frame. While by the symbol $\alpha \in \mu$ we denote Young frames $\alpha \vdash n-k$ obtained from a Young frame $\mu \vdash n$ by subtracting $k$ boxes. Such a procedure can be performed on many different ways, and total number of them we denote as $m_{\mu / \alpha}$. In the general situation we do not have closed expression for computing $m_{\mu / \alpha}$, however in the qubit case (Young frames up to two rows) we have [1? , 2]

$$
m_{\mu / \alpha}=k ! \operatorname{det}\left(\frac{1}{\alpha_{i}-\mu_{j}-i+j}\right)_{i, j=1,2} .
$$

Having the above description of all the necessary objects and facts, we are in position to prove expressions for the entanglement fidelity $F$ and averaged probability of success $p_{\text {succ }}$ form the main text. In general, for qudits, the formulas are expressed in terms of:

i) dimensionality $d_{\mu}$ of the irrep $\mu$

ii) its multiplicity $m_{\mu}$ in the representation of $S(n)$ permuting $n$ qdits.

iii) numbers $m_{\mu / \alpha}$ describing number of possibilities of obtaining a frame $\mu \vdash n$ from a frame $\alpha \vdash n-k$ by adding $k$ boxes,

and have the following form for the entanglement fidelity [30]:

$$
F=\frac{1}{d^{N+2 k}} \sum_{\alpha \vdash N-k}\left(\sum_{\mu \in \alpha} m_{\mu / \alpha} \sqrt{m_{\mu} d_{\mu}}\right)^{2} .
$$




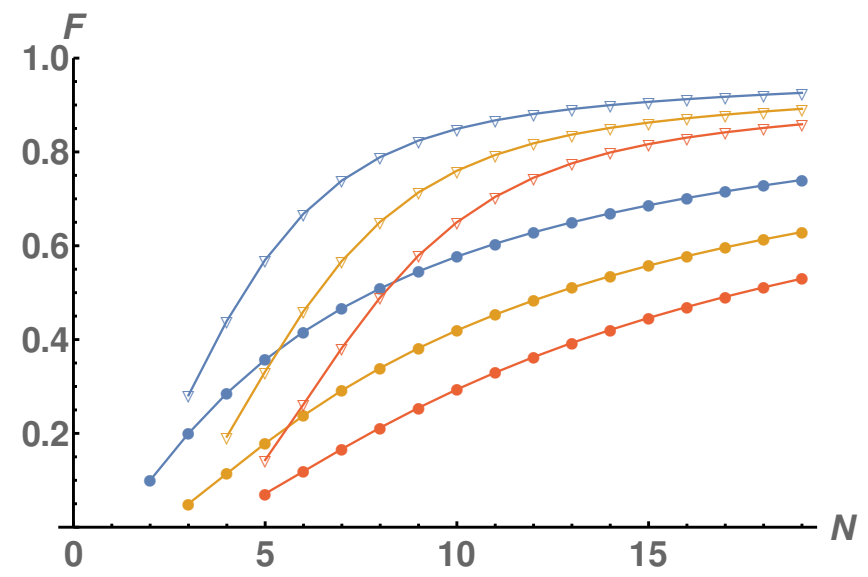

Figure 8: Comparison of the first bound (9) on entanglement fidelity from Theorem 2 in the main text (full circles (full circles, for $k=2$ marked with $\bullet, k=3 \bullet, k=4 \bullet)$ ) with exact values for the qubit case (reversed triangle markers, for $k=2$ marked with $\nabla, k=3 \nabla, k=4 \nabla)$.

and the averaged probability of success [30]:

$$
p_{\text {succ }}=\frac{1}{d^{N}} \sum_{\alpha \vdash N-k} m_{\alpha}^{2} \min _{\mu \in \alpha} \frac{d_{\mu}}{m_{\mu}} .
$$

For qubits we shall see that these quantities are expressed in more familiar language of angular momentum. Having a Young frame $\alpha \vdash N-k$ we denote the Young frame $\mu \vdash N$ obtained from $\alpha$ by adding consecutive $k$ boxes by $\mu \in \alpha$. The number of such allowed additions is $m_{\mu / \alpha}$. The multiplicity $m_{\mu}$ equals dimension of spin- $j$ representation of $S U(2)^{\otimes(N-k)}$, where $2 j=\mu_{1}-\mu_{2}$ and thus $m_{\mu}=2 j+1$; the dimension $d_{\mu}$ corresponds to multiplicity of spin- $j$ representation, given by $\frac{(2 j+1)(N-k) !}{((N-k) / 2+j+1) !((N-k) / 2-j) !}$. Finally, expression (64) can be expressed as

$$
m_{2 s, 2 j, k}=\left(\begin{array}{c}
k \\
s-j+k / 2
\end{array}\right)-\left(\begin{array}{c}
k \\
s+j+k / 2+1
\end{array}\right) .
$$

Thus, considering that spin of $N-k$ spin- $\frac{1}{2}$ particles can take values from 0 or $1 / 2$ depending on the parity of $N-k$ to $\frac{N-k}{2}$ and after adding $k$ particles either from $0\left(\frac{1}{2}\right)$ or $s-k / 2$, whichever quantity is greater, to $s+k / 2$, We can rewrite expression (65) as

$$
F=\frac{1}{2^{N+2 k}} \sum_{s=0\left(\frac{1}{2}\right)}^{\frac{N-k}{2}}\left(\sum_{j=\max \left\{0\left(\frac{1}{2}\right), s-\frac{k}{2}\right\}}^{s+\frac{k}{2}}\left(\left(\begin{array}{c}
k \\
s-j+k / 2
\end{array}\right)-\left(\begin{array}{c}
k \\
s+j+k / 2+1
\end{array}\right)\right) \sqrt{\frac{(2 j+1)^{2} N !}{(N / 2-j) !(N / 2+j+1) !}}\right)^{2} .
$$

Defining auxiliary quantity $h_{s j k}$ of the form

$$
h_{s j k}=\left(\begin{array}{c}
k \\
s-j+k / 2
\end{array}\right)-\left(\begin{array}{c}
k \\
s+j+k / 2+1
\end{array}\right),
$$

and simplifying expression under the square root, we arrive to the final formula for the fidelity:

$$
F=\frac{1}{2^{N+2 k}} \sum_{s=0\left(\frac{1}{2}\right)}^{\frac{N-k}{2}}\left(\sum_{j=\max \left\{0\left(\frac{1}{2}\right), s-\frac{k}{2}\right\}}^{s+\frac{k}{2}} \frac{2 j+1}{N+1} \sqrt{\left(\begin{array}{l}
N+1 \\
\frac{N}{2}-j
\end{array}\right)} h_{s j k}\right)^{2}
$$

On Figure 8 we show comparison of the first bound (9) from Theorem 2 of the main text (Appendix B) with the exact expression from expression (70).

In case of probability of success, using directly the argumentation from Section V, we rewrite expression (66) as:

$$
p_{\text {succ }}(N, k)=\frac{1}{2^{N}} \sum_{s=0\left(\frac{1}{2}\right)}^{\frac{N-k}{2}} \min _{j} \frac{N !(2 s+1)^{2}}{(N / 2-j) !(N / 2+j+1) !}=\frac{1}{2^{N}} \sum_{s=0\left(\frac{1}{2}\right)}^{\frac{N-k}{2}} \frac{N !(2 s+1)^{2}}{(N / 2-s-k / 2) !(N / 2+s+k / 2+1) !} .
$$


Observing that

$$
\frac{N !}{(N / 2-s-k / 2) !(N / 2+s+k / 2+1) !}=\frac{1}{N+1}\left(\begin{array}{c}
N+1 \\
\frac{N-k}{2}-s
\end{array}\right) .
$$

we get the final form given in by the following equation

$$
p_{\text {succ }}(N, k)=\frac{1}{2^{N}} \sum_{s=0\left(\frac{1}{2}\right)}^{\frac{N-k}{2}} \frac{1}{N+1}\left(\begin{array}{c}
N+1 \\
\frac{N-k}{2}-s
\end{array}\right)
$$

In Section D of this appendix we discuss additional properties of probability of success $p_{\text {succ }}$.

\section{Bounds for finite number of ports $N$}

To achieve our goals in this section we have combined in a non-trivial way advanced tools emerging from statistical analysis. Motivated by Berry-Essen Theorem [6, 10], which quantifies the rate of convergence to normal distribution, together with Central Limit Theorem type reasoning we express probability of success in terms of Gaussian integrals. In particular, starting from expression (73) in the finite case, we provide useful lower and upper bounds on probability of success of the perfect transmission in MPBT protocol. We can observe, that it closely resembles the expression for the probability distribution function of the distribution of number of successes in $N+1$ Bernoulli trials each with $p=1 / 2$. We start from proving the following:

Proposition 18. Let $k=a \sqrt{N}, a \in(0,2)$. Then

$2\left(\int_{0}^{\infty} x^{2} \frac{1}{\sqrt{2 \pi}} \mathrm{e}^{-\frac{\left(x+a \sqrt{\frac{N}{N+1}}\right)^{2}}{2}} d x-M(N)-I_{1}^{B}(N)-I_{2}^{B}(N)\right)-\delta^{B}(N) \leq p_{\text {succ }} \leq 2\left(\int_{0}^{\infty} x^{2} \frac{1}{\sqrt{2 \pi}} \mathrm{e}^{-\frac{\left(x+a \sqrt{\frac{N}{N+1}}\right)^{2}}{2}} d x+M(N)\right.$

where

$$
\begin{aligned}
& M(N)=\frac{2}{\mathrm{e} \sqrt{N+1}} \frac{1}{\sqrt{2 \pi}}, \quad I_{1}^{B}(N)=(N+1)^{-\frac{3}{2}} \frac{1}{\sqrt{2 \pi}}, \\
& I_{2}^{B}(N)=\left(\frac{\sqrt{N+1}}{\sqrt{2 \pi}}+1\right) \mathrm{e}^{-\frac{(\sqrt{N+1}-1)^{2}}{2}}, \\
& \delta^{B}(N)=4 N^{-1 / 4}+11 \sqrt{N+1} \mathrm{e}^{-\frac{\left(N^{5 / 8}-1\right)^{2}}{N+1}} .
\end{aligned}
$$

The reasoning above applies to finite number of ports. We can see the convergence of these bounds in Figure 9 Taking the limit $N \rightarrow \infty$ we can formulate the following

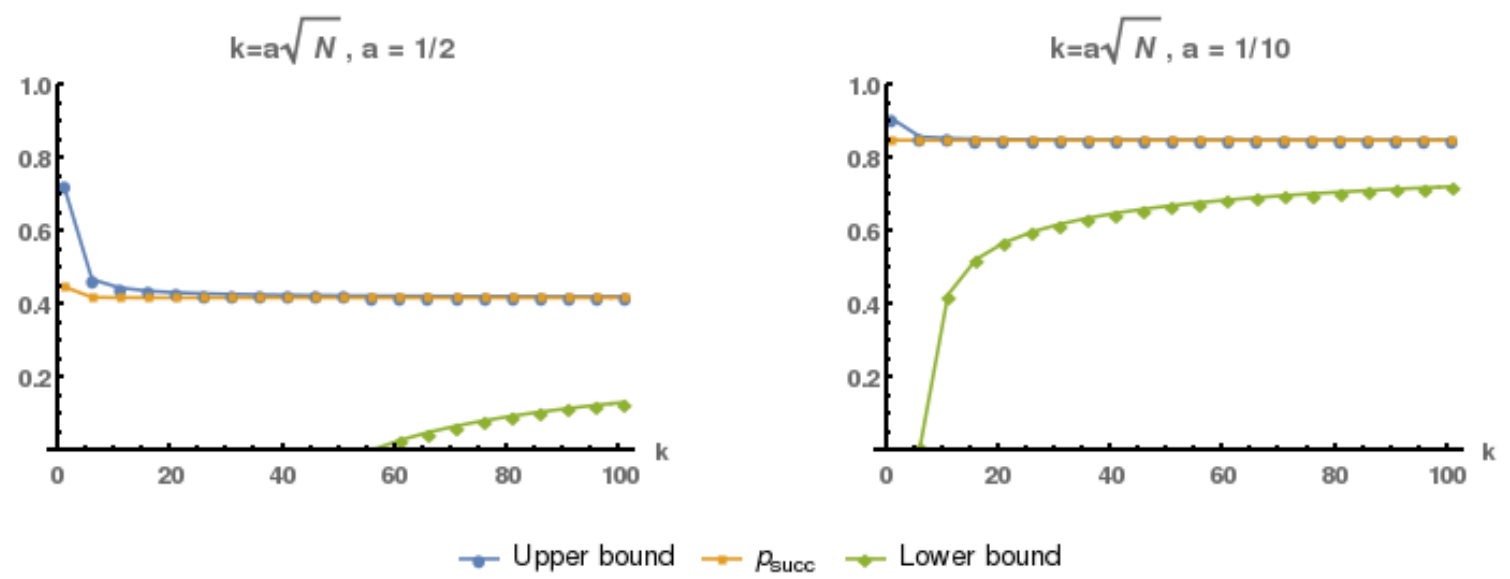

Figure 9: The figures present lower and upper bounds for $p_{\text {succ }}$ given in Proposition 18, for two different values of $a$, where the number of teleported qubits is given by $k=a \sqrt{N}$. 
Corollary 19. The limiting value of $p_{\text {succ }}$, where $k=a \sqrt{N}, a \in(0,1), N \rightarrow \infty$ is

$$
\lim _{N \rightarrow \infty} p_{\text {succ }}=2 \int_{0}^{\infty} x^{2} \frac{1}{\sqrt{2 \pi}} \mathrm{e}^{-\frac{(x+a)^{2}}{2}} d x .
$$

When we take $k=o(\sqrt{N})$, i.e. $k / \sqrt{N} \rightarrow 0$ when $N \rightarrow 0$, we can apply the bounds (131)-(133) substituting $a \sqrt{\frac{N}{N+1}}$ with $k / \sqrt{N}$, and when we take the limit $N \rightarrow \infty$ we obtain

Corollary 20. When $k=o(\sqrt{N})$ then the limit of $p_{\text {succ }}$ is

$$
\lim _{N \rightarrow \infty} p_{\text {succ }}=2 \int_{0}^{\infty} x^{2} \frac{1}{\sqrt{2 \pi}} \mathrm{e}^{-\frac{x^{2}}{2}} d x=1
$$

Proof of Proposition 18. The probability of success in the multi-port teleportation scheme, for even $N$ can be written as:

$$
p_{s}=\frac{1}{N+1} \sum_{s=0}^{\frac{N-k}{2}}(2 s+1)^{2} \operatorname{Pr}\left(X=\frac{N-k}{2}-s\right) .
$$

Instead of using discrete probability distribution $\operatorname{Pr}(X \leq l)$ we can approximate it by Gaussian distribution $\Phi\left(\frac{l-\frac{N+1}{2}}{\frac{1}{2} \sqrt{N+1}}\right)$. The error of such estimation is known due to the Berry-Essen Theorem [6, 10], which in our case reads as

$$
\forall l=0, \ldots, N+1 \quad\left|\operatorname{Pr}(X \leq l)-\Phi\left(\frac{l-\frac{N+1}{2}}{\frac{1}{2} \sqrt{N+1}}\right)\right| \leq \frac{4 c}{\sqrt{N+1}}
$$

The real constant $c$ can be analytically bounded from below and due to [11] there is $c \geq \frac{\sqrt{10}+3}{6 \sqrt{2 \pi}} \approx 0.4097$. In this paper for our purposes we set $c=1 / 2$. We can rewrite $\Phi\left(\frac{l-\frac{N+1}{2}}{\frac{1}{2} \sqrt{N+1}}\right)$ as

$$
\Phi\left(\frac{l-\frac{N+1}{2}}{\frac{1}{2} \sqrt{N+1}}\right)=\int_{-\infty}^{-\frac{N-1}{2}} G\left(0, \sigma=\frac{1}{2} \sqrt{N+1} ; x\right) \mathrm{d} x+\sum_{i=0}^{l} \int_{-\frac{N+i}{2}}^{-\frac{N+i}{2}+1} G\left(0, \frac{1}{2} \sqrt{N+1} ; x\right) \mathrm{d} x
$$

The first term of the above expression decays to 0 for $N \rightarrow \infty$, more precisely we have

$$
\begin{aligned}
\int_{-\infty}^{-\frac{N-1}{2}} G\left(0, \sigma=\frac{1}{2} \sqrt{N+1} ; x\right) \mathrm{d} x & =\frac{2}{\sqrt{2 \pi(N+1)}} \int_{-\infty}^{-\frac{N-1}{2}} \exp \left(-\frac{2 x^{2}}{N+1}\right) \mathrm{d} x \\
& =\frac{2}{\sqrt{2 \pi(N+1)}} \int_{\frac{N-1}{2}}^{\infty} \exp \left(-\frac{2 x^{2}}{N+1}\right) \mathrm{d} x \\
& \leq \sqrt{\frac{N+1}{2}} \exp \left(-\frac{N+1}{2}\right):=G_{1}(N),
\end{aligned}
$$

since the Hoeffding bound states

$$
\int_{r}^{\infty} \exp \left(-\widetilde{c}^{2} x^{2}\right) \mathrm{d} x \leq \frac{\sqrt{\pi}}{\widetilde{c}} \exp \left(-\widetilde{c}^{2} r^{2}\right) .
$$

Notice that factor $G_{1}(N)$ decays to 0 for $N \rightarrow \infty$. Now, due to $(81)$, we have $\forall l=0, \ldots, N+1$

$$
\begin{aligned}
\left|\operatorname{Pr}(X \leq l)-\Phi\left(\frac{l-\frac{N+1}{2}}{\frac{1}{2} \sqrt{N+1}}\right)\right| & \leq\left|\operatorname{Pr}(X \leq l)-\sum_{i=0}^{l} \int_{-\frac{N+i}{2}}^{-\frac{N+i}{2}+1} G\left(0, \frac{1}{2} \sqrt{N+1} ; x\right) \mathrm{d} x\right|+G_{1}(N) \\
& =\left|\sum_{i=0}^{l} \operatorname{Pr}(X=i)-\sum_{i=0}^{l} \int_{-\frac{N+i}{2}}^{-\frac{N+i}{2}+1} G\left(0, \frac{1}{2} \sqrt{N+1} ; x\right) \mathrm{d} x\right|+G_{1}(N) \\
& =\sum_{i=0}^{l}\left|\operatorname{Pr}(X=i)-\int_{-\frac{N+i}{2}}^{-\frac{N+i}{2}+1} G\left(0, \frac{1}{2} \sqrt{N+1} ; x\right) \mathrm{d} x\right|+G_{1}(N) .
\end{aligned}
$$


Getting back to probability of success in (78):

$$
\begin{aligned}
\frac{2}{N+1} \sum_{s=0}^{\frac{N-k}{2}}(2 s+1)^{2} \Phi_{\sigma} & \left(-\frac{k}{2}-s-\frac{1}{2},-\frac{k}{2}-s+\frac{1}{2}\right) \geq \\
p_{s} & =\frac{2}{N+1} \sum_{s=0}^{\frac{N-k}{2}}(2 s+1)^{2} \operatorname{Pr}\left(X=\frac{N-k}{2}-s\right) \\
\geq & \frac{2}{N+1}\left(\sum_{s=0}^{\frac{N-k}{2}}(2 s+1)^{2} \Phi_{\sigma}\left(-\frac{k}{2}-s-\frac{1}{2},-\frac{k}{2}-s+\frac{1}{2}\right)-\sum_{s=0}^{\frac{N-k}{2}}(2 s+1)^{2} \delta_{s}\right),
\end{aligned}
$$

where

$$
\delta_{s}=\left|\operatorname{Pr}\left(x=\frac{N-k}{2}-s\right)-\Phi_{\sigma}\left(-\frac{k}{2}-s-\frac{1}{2},-\frac{k}{2}-s+\frac{1}{2}\right)\right|, \quad \sigma=\frac{1}{2} \sqrt{N+1} .
$$

The upper bound comes from the Lemma 7.2 from [26]. Since $X \sim B\left(N+1, \frac{1}{2}\right)$

$$
\Phi_{\sigma}\left(-\frac{k}{2}-s-\frac{1}{2},-\frac{k}{2}-s+\frac{1}{2}\right) \geq \operatorname{Pr}\left(X=\frac{N-k}{2}-s\right)
$$

if

$$
\frac{N+1}{2}+1 \leq N+1-\left(\frac{N-k}{2}-s\right)
$$

which is equivalent to $k+2 s \geq 1$ which clearly holds for $k$ and $s$ in question.

At this point we can divide further considerations into two main steps:

1. Showing that $\frac{2}{N+1} \sum_{s=0}^{\frac{N-k}{2}}(2 s+1)^{2} \delta_{s} \rightarrow 0$ for $N \rightarrow \infty$ in (84).

2. Evaluating the first term $\frac{2}{N+1} \sum_{s=0}^{\frac{N-k}{2}}(2 s+1)^{2} \Phi_{\sigma}\left(-\frac{k}{2}-s-\frac{1}{2},-\frac{k}{2}-s+\frac{1}{2}\right)$ from expression (84) in the asymptotic regime $N \rightarrow \infty$.

Showing decaying of the second term in (84).

Let us focus on the first point. We write

$$
\frac{2}{N+1} \sum_{s=0}^{\frac{N-k}{2}}(2 s+1)^{2} \delta_{s}=\underbrace{\frac{2}{N+1} \sum_{s=0}^{m(N)}(2 s+1)^{2} \delta_{s}}_{A}+\underbrace{\frac{2}{N+1} \sum_{s=m(N)}^{\frac{N-k}{2}}(2 s+1)^{2} \delta_{s}}_{B} .
$$

Substituting $m(N)=(1 / 2)(b(N) \sqrt{N}-1)$, using fact $\sum_{s=0}^{\frac{N-k}{2}} \delta_{s} \leq \frac{4 c}{\sqrt{N+1}}$ with $c=1 / 2$, we bound $A$ as

$$
A \leq \frac{2(2 m(N)+1)^{2}}{N+1} \sum_{s=0}^{m(N)} \delta_{s} \leq \frac{4 b^{2}(N) N}{(N+1)^{3 / 2}} \leq \frac{4 N b^{2}(N)}{N^{3 / 2}}=4 N^{-1 / 4}
$$

where we choose for example $b(N)=N^{1 / 8}$ getting demanded asymptotic. For the factor $B$, first we write

$$
\begin{aligned}
\delta_{s} & \leq \operatorname{Pr}\left(x=\frac{N-k}{2}-s\right)+\Phi_{\sigma}\left(-\frac{k}{2}-s-\frac{1}{2},-\frac{k}{2}-s+\frac{1}{2}\right) \\
& \leq \operatorname{Pr}\left(x \leq \frac{N-k}{2}-s\right)+\Phi_{\sigma}\left(-\frac{k}{2}-s+\frac{1}{2}\right) \\
& \leq \exp \left(-\frac{(k+2 s+1)^{2}}{2(N+1)}\right)+\exp \left(-\frac{(k+2 s-1)^{2}}{2(N+1)}\right) \\
& \leq 2 \exp \left(-\frac{(k+2 s-1)^{2}}{2(N+1)}\right) .
\end{aligned}
$$


Indeed, for $\Phi_{\sigma}\left(-\frac{k}{2}-s+\frac{1}{2}\right)$ we use $\sigma=\frac{1}{2} \sqrt{N+1}$ and the Hoeffding bound (82):

$$
\begin{aligned}
\Phi_{\sigma}\left(-\frac{k}{2}-s+\frac{1}{2}\right)=\frac{1}{\sqrt{2 \pi} \sigma} \int_{-\frac{k}{2}-s+\frac{1}{2}}^{\infty} \exp \left(-\frac{1}{2} \frac{x^{2}}{\sigma^{2}}\right) \mathrm{d} x & \leq \exp \left(-\frac{1}{2} \frac{\left(-\frac{k}{2}-s+\frac{1}{2}\right)^{2}}{\sigma^{2}}\right) \\
& =\exp \left(-\frac{(k+2 s-1)^{2}}{2(N+1)}\right),
\end{aligned}
$$

For $\operatorname{Pr}\left(x \leq \frac{N-k}{2}-s\right)$ we use the Chernoff bound $\operatorname{Pr}(X<(1-\delta) \mu) \leq \mathrm{e}^{-\frac{\mu \delta^{2}}{2}}$, with $\mu=N+1$ and $1-\delta=\frac{N-k-2 s}{N+1}$ :

$$
\operatorname{Pr}\left(x \leq \frac{N-k}{2}-s\right) \leq \exp \left(-\frac{(k+2 s+1)^{2}}{2(N+1)}\right) .
$$

Applying (90) to $B$ we reduce to

$$
\begin{aligned}
B \leq \frac{4}{N+1} \sum_{s=m(N)}^{\frac{N-k}{2}}(2 s+1)^{2} \exp \left(-\frac{(k+2 s-1)^{2}}{2(N+1)}\right) & \leq \frac{4(N-k+1)}{N+1} \sum_{s=m(N)}^{\frac{N-k}{2}} \exp \left(-\frac{(k+2 s-1)^{2}}{2(N+1)}\right) \\
& \leq \frac{4(N-k+1)}{N+1} \sum_{s=m(N)}^{\infty} \exp \left(-\frac{(k+2 s-1)^{2}}{2(N+1)}\right) \\
& \leq 4 \sum_{s=m(N)}^{\infty} \exp \left(-\frac{(k+2 s-1)^{2}}{2(N+1)}\right) \\
& \leq \frac{11}{\sqrt{N+1}} \exp \left(-\frac{(k+2 m(N)-1)^{2}}{2(N+1)}\right) \\
& \leq 11 \sqrt{N+1} \exp \left(-\frac{2 m^{2}(N)}{N+1}\right),
\end{aligned}
$$

where in the fourth inequality we use the Hoeffding inequality. Finally, using explicit form of the function $m(N)=(1 / 2)\left(N^{5 / 8}-1\right)$ and expression (89) we bound left-hand side of (88) as

$$
\frac{2}{N+1} \sum_{s=0}^{\frac{N-k}{2}}(2 s+1)^{2} \delta_{s} \leq 4 N^{-3 / 2}+11 \sqrt{N+1} \exp \left(-\frac{\left(N^{5 / 8}-1\right)^{2}}{N+1}\right)
$$

which clearly decays to 0 with $N \rightarrow \infty$. It means $p_{s}$ in (84) can be bounded from the below

$$
p_{s} \geq \frac{2}{N+1} \sum_{s=0}^{\frac{N-k}{2}}(2 s+1)^{2} \Phi_{\sigma}\left(-\frac{k}{2}-s-\frac{1}{2},-\frac{k}{2}-s+\frac{1}{2}\right)-4 N^{-1 / 4}-11 \sqrt{N+1} \exp \left(-\frac{\left(N^{5 / 8}-1\right)^{2}}{N+1}\right) .
$$

Estimation of the first term in (84). We now want to estimate the expression

$$
\frac{2}{N+1}\left(\sum_{s=0}^{\frac{N-k}{2}}(2 s+1)^{2} \Phi_{\sigma}\left(-\frac{k}{2}-s-\frac{1}{2},-\frac{k}{2}-s+\frac{1}{2}\right)\right) .
$$

First, we can write

$$
\begin{aligned}
& \frac{2}{N+1}\left(\sum_{s=0}^{\frac{N-k}{2}}(2 s+1)^{2} \Phi_{\sigma}\left(-\frac{k}{2}-s-\frac{1}{2},-\frac{k}{2}-s+\frac{1}{2},\right)\right)=\frac{2}{N+1} \sum_{s=0}^{\frac{N-k}{2}}(2 s+1)^{2} \int_{-\frac{k}{2}-s-\frac{1}{2}}^{-\frac{k}{2}-s+\frac{1}{2}} \frac{2}{\sqrt{2 \pi(N+1)}} \mathrm{e}^{-\frac{2 x^{2}}{N+1}} d x \\
& \geq \frac{2}{N+1} \sum_{s=0}^{\frac{N-k}{2}}(2 s+1)^{2} \frac{2}{\sqrt{2 \pi(N+1)}} \mathrm{e}^{-\frac{(k+2 s+1)^{2}}{2(N+1)}} \\
& =2 \frac{2}{\sqrt{N+1}} \sum_{s=0}^{\frac{N-k}{2}}\left(\frac{1}{\sqrt{N+1}}+s \frac{2}{\sqrt{N+1}}\right)^{2} \frac{1}{\sqrt{2 \pi}} \mathrm{e}^{-\frac{\left(\frac{1}{\sqrt{N+1}}+s \frac{2}{\sqrt{N+1}}+\frac{k}{\sqrt{N+1}}\right)^{2}}{2}}
\end{aligned}
$$


where we replace integral over interval $\left[-\frac{k}{2}-s-\frac{1}{2},-\frac{k}{2}-s+\frac{1}{2}\right]$ with local minimum of integrated function multiplied by the length of the interval, which is 1 . One can identify the last expression as a Riemann left-sum of function

$$
f^{N}(z):=z^{2} \mathrm{e}^{\frac{\left(z+\frac{k}{\sqrt{N+1}}\right)^{2}}{2}}
$$

on the interval $\left[\frac{1}{\sqrt{N+1}}, \frac{N-k}{\sqrt{N+1}}\right]$. We will transform this sum in the manner depicted in Figure 10 . Setting $k=a \sqrt{N}$ the target function takes form

$$
f^{N}(z)=z^{2} \mathrm{e}^{\frac{\left(z+a \sqrt{\frac{N}{N+1}}\right)^{2}}{2}}
$$

One can check, that for

$$
0 \leq z \leq m:=\frac{-\sqrt{\frac{N}{N+1}} a+\sqrt{\frac{N}{N+1} a^{2}+8}}{2}
$$

it is monotonically increasing, while for $z \geq m$ it decreases. Therefore it can be split into two components: one consisting of partitions $\left\{\left[\frac{1}{\sqrt{N+1}}, \frac{1+2 s}{\sqrt{N+1}}\right], s \in 0, \ldots, s_{m}\right\}$ where $\frac{1+2 s_{m}}{\sqrt{N+1}} \leq m$, and the other consisting of partitions for higher values of $s$. Thus we have

$$
\begin{aligned}
\frac{2}{\sqrt{N+1}} \sum_{s=0}^{\frac{N-k}{2}} & \left(\frac{1}{\sqrt{N+1}}+s \frac{2}{\sqrt{N+1}}\right)^{2} \frac{1}{\sqrt{2 \pi}} \mathrm{e}^{-\frac{\left(\frac{1}{\sqrt{N+1}}+s \frac{2}{\sqrt{N+1}}+\frac{k}{\sqrt{N+1}}\right)^{2}}{2}} \\
= & 2 \frac{2}{\sqrt{N+1}} \sum_{s=0}^{s_{m}}\left(\frac{1}{\sqrt{N+1}}+s \frac{2}{\sqrt{N+1}}\right)^{2} \frac{1}{\sqrt{2 \pi}} \mathrm{e}^{-\frac{\left(\frac{1}{\sqrt{N+1}}+s \frac{2}{\sqrt{N+1}}+\frac{k}{\sqrt{N+1}}\right)^{2}}{2}} \\
& +2 \frac{2}{\sqrt{N+1}} \sum_{s=s_{m}+1}^{\frac{N-k}{2}}\left(\frac{1}{\sqrt{N+1}}+s \frac{2}{\sqrt{N+1}}\right)^{2} \frac{1}{\sqrt{2 \pi}} \mathrm{e}^{\left.-\frac{\left(\frac{1}{\sqrt{N+1}}+s \frac{2}{\sqrt{N+1}}+\frac{k}{\sqrt{N+1}}\right.}{2}\right)^{2}} \\
= & S_{L}^{N}\left(\frac{1}{\sqrt{N+1}}, \frac{1+2\left(s_{m}+1\right)}{\sqrt{N+1}}\right)+S_{L}^{N}\left(\frac{1+2\left(s_{m}+1\right)}{\sqrt{N+1}}, \frac{N-a \sqrt{N}+1}{\sqrt{N+1}}\right),
\end{aligned}
$$

where $S_{L}^{N}\left(\frac{1}{\sqrt{N+1}}, \frac{1+2\left(s_{m}+1\right)}{\sqrt{N+1}}\right)$ denotes left Riemann sum on the interval $\left[\frac{1}{\sqrt{N+1}}, \frac{1+\left(2 s_{m}+1\right)}{\sqrt{N+1}}\right]$. Setting $t=s+1$, we can write

$$
\begin{aligned}
S_{L}^{N}\left(\frac{1}{\sqrt{N+1}}, \frac{2 s_{m}+1}{\sqrt{N+1}}\right) & =2 \frac{2}{\sqrt{N+1}} \sum_{s=0}^{s_{m}}\left(\frac{1}{\sqrt{N+1}}+s \frac{2}{\sqrt{N+1}}\right)^{2} \frac{1}{\sqrt{2 \pi}} \mathrm{e}^{-\frac{\left(\frac{1}{\sqrt{N+1}}+s \frac{2}{\sqrt{N+1}}+\frac{k}{\sqrt{N+1}}\right)^{2}}{2}} \\
& =2 \frac{2}{\sqrt{N+1}} \sum_{t=1}^{t_{m}}\left(-\frac{1}{\sqrt{N+1}}+t \frac{2}{\sqrt{N+1}}\right)^{2} \frac{1}{\sqrt{2 \pi}} \mathrm{e}^{-\frac{\left(-\frac{1}{\sqrt{N+1}}+t \frac{2}{\sqrt{N+1}}+\frac{k}{\sqrt{N+1}}\right)^{2}}{2}} \\
& =S_{R}^{N}\left(-\frac{1}{\sqrt{N+1}}, \frac{-1+t_{m}}{\sqrt{N+1}}\right) .
\end{aligned}
$$

Thus

$$
\begin{aligned}
& S_{L}^{N}\left(\frac{1}{\sqrt{N+1}}, \frac{1+2\left(s_{m}+1\right)}{\sqrt{N+1}}\right)+S_{L}^{N}\left(\frac{1+2\left(s_{m}+1\right)}{\sqrt{N+1}}, \frac{N-a \sqrt{N}+1}{\sqrt{N+1}}\right) \\
& =S_{R}^{N}\left(-\frac{1}{\sqrt{N+1}}, \frac{-1+2 t_{m}}{\sqrt{N+1}}\right)+S_{L}^{N}\left(\frac{1+2\left(s_{m}+1\right)}{\sqrt{N+1}}, \frac{N-a \sqrt{N}+1}{\sqrt{N+1}}\right) .
\end{aligned}
$$

We can observe, that we shifted the first left sum by $-\frac{2}{\sqrt{N+1}}$, which corresponds to single partition interval, turning it into right sum. Supplementing the upper bound of the "missing middle term" $\frac{2}{\sqrt{N+1}} m^{2} \frac{1}{\sqrt{2 \pi}} \mathrm{e}^{-\frac{\left(m+\frac{k}{\sqrt{N+1}}\right)^{2}}{2}}$, 
we obtain the following restriction

$$
\begin{aligned}
S_{R}^{N}\left(-\frac{1}{\sqrt{N+1}}, \frac{-1+2 t_{m}}{\sqrt{N+1}}\right)+S_{L}^{N}\left(\frac{1+2\left(s_{m}+1\right)}{\sqrt{N+1}}, \frac{N-a \sqrt{N}+1}{\sqrt{N+1}}\right)+\frac{2}{\sqrt{N+1}} m^{2} \frac{1}{\sqrt{2 \pi}} \mathrm{e}^{-\frac{\left(m+\frac{k}{\sqrt{N+1}}\right)^{2}}{2} d x} \\
\geq \int_{-\frac{1}{\sqrt{N+1}}}^{\frac{N-a \sqrt{N}+1}{\sqrt{N+1}}} x^{2} \frac{1}{\sqrt{2 \pi}} \mathrm{e}^{-\frac{\left(x+a \sqrt{\frac{N}{N+1}}\right)^{2}}{2} d x} \\
\geq \int_{\frac{1}{\sqrt{N+1}}}^{\frac{N-a \sqrt{N+1}}{\sqrt{N+1}}} x^{2} \frac{1}{\sqrt{2 \pi}} \mathrm{e}^{-\frac{\left(x+a \sqrt{\frac{N}{N+1}}\right)^{2}}{2} d x .}
\end{aligned}
$$

These three sums are depicted in Figure 10.
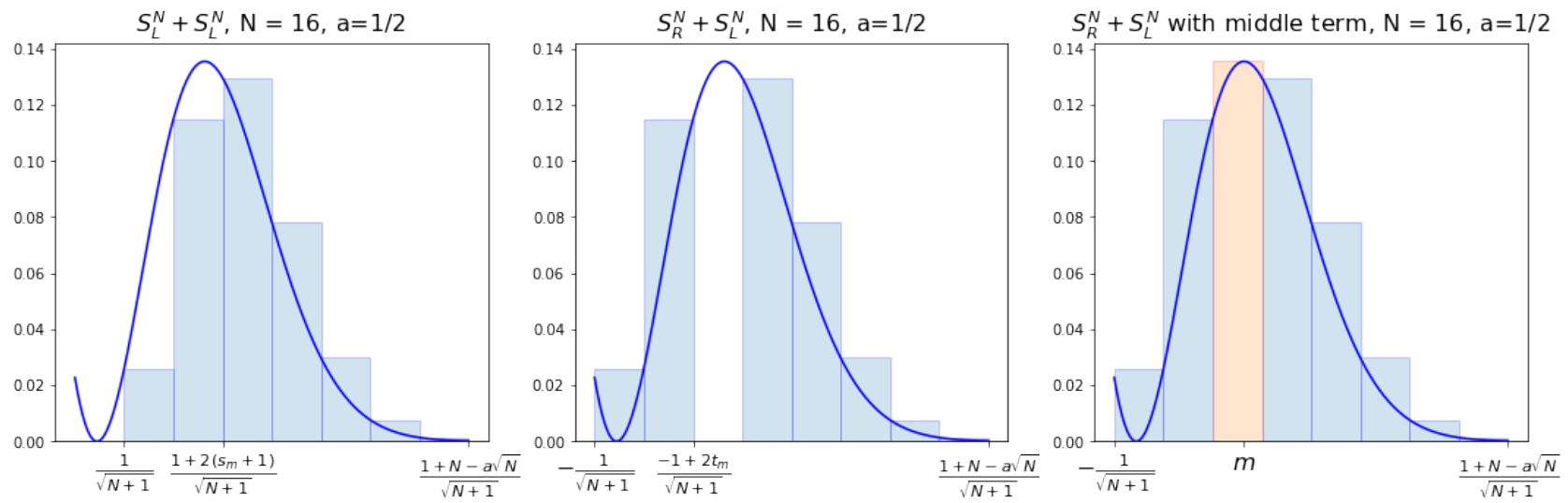

Figure 10: Three Riemann sums: (110), (111) and (112).

Thus the initial Riemann sum (110) is bounded by

$$
\begin{aligned}
& \frac{2}{\sqrt{N+1}} \sum_{s=0}^{\frac{N-k}{2}}\left(\frac{1}{\sqrt{N+1}}+s \frac{2}{\sqrt{N+1}}\right)^{2} \frac{1}{\sqrt{2 \pi}} \mathrm{e}^{-\frac{\left(\frac{1}{\sqrt{N+1}}+s \frac{2}{\sqrt{N+1}}+\frac{k}{\sqrt{N+1}}\right)^{2}}{2}} \\
& =S_{L}^{N}\left(\frac{1}{\sqrt{N+1}}, \frac{2 s_{m}+3}{\sqrt{N+1}}\right)+S_{L}^{N}\left(\frac{2 s_{m}+3}{\sqrt{N+1}}, \frac{N-a \sqrt{N}+1}{\sqrt{N+1}}\right) \\
& =S_{R}^{N}\left(-\frac{1}{\sqrt{N+1}}, \frac{2 s_{m}+1}{\sqrt{N+1}}\right)+S_{L}^{N}\left(\frac{2 s_{m}+3}{\sqrt{N+1}}, \frac{N-a \sqrt{N}+1}{\sqrt{N+1}}\right) \\
& \geq \int_{\frac{1}{\sqrt{N+1}}}^{\frac{N-a \sqrt{N}+1}{\sqrt{N+1}}} x^{2} \frac{1}{\sqrt{2 \pi}} \mathrm{e}^{-\frac{\left(x+a \sqrt{\frac{N}{N+1}}\right)^{2}}{2}} d x-\frac{2}{\sqrt{N+1}} m^{2} \frac{1}{\sqrt{2 \pi}} \mathrm{e}^{\frac{\left(m+\frac{k}{\sqrt{N+1}}\right)^{2}}{2}} \\
& \geq \int_{\frac{1}{\sqrt{N+1}}}^{\frac{N-a \sqrt{N}+1}{\sqrt{N+1}}} x^{2} \frac{1}{\sqrt{2 \pi}} \mathrm{e}^{-\frac{\left(x+a \sqrt{\frac{N}{N+1}}\right)^{2}}{2}} d x-\frac{2}{\sqrt{N+1}} m^{2} \frac{1}{\sqrt{2 \pi}} \mathrm{e}^{\frac{m^{2}}{2}} \\
& \geq \int_{\frac{1}{\sqrt{N+1}}}^{\frac{N-a \sqrt{N}+1}{\sqrt{N+1}}} x^{2} \frac{1}{\sqrt{2 \pi}} \mathrm{e}^{-\frac{\left(x+a \sqrt{\frac{N}{N+1}}\right)^{2}}{2}} d x-\frac{4}{\mathrm{e} \sqrt{N+1}} \frac{1}{\sqrt{2 \pi}} \\
& \geq \int_{\frac{1}{\sqrt{N+1}}}^{\frac{N-a \sqrt{N}+1}{\sqrt{N+1}}} x^{2} \frac{1}{\sqrt{2 \pi}} \mathrm{e}^{-\frac{\left(x+a \sqrt{\frac{N}{N+1}}\right)^{2}}{2}} d x-\frac{2}{\sqrt{N+1}} \frac{1}{\sqrt{2 \pi}}
\end{aligned}
$$


and thus (96) admits the following restriction

$$
\begin{aligned}
& \frac{2}{N+1}\left(\sum_{s=0}^{\frac{N-k}{2}}(2 s+1)^{2} \Phi_{\sigma}\left(-\frac{k}{2}-s-\frac{1}{2},-\frac{k}{2}-s+\frac{1}{2}\right)\right) \\
& \quad \geq 2 \frac{2}{\sqrt{N+1}} \sum_{s=0}^{\frac{N-k}{2}}\left(\frac{1}{\sqrt{N+1}}+s \frac{2}{\sqrt{N+1}}\right)^{2} \frac{1}{\sqrt{2 \pi}} \mathrm{e}^{-\frac{\left(\frac{1}{\sqrt{N+1}}+s \frac{2}{\sqrt{N+1}}+\frac{k}{\sqrt{N+1}}\right)^{2}}{2}} \\
& \quad \geq 2\left(\int_{\frac{1}{\sqrt{N+1}}}^{\frac{N-a \sqrt{N+1}}{\sqrt{N+1}}} x^{2} \frac{1}{\sqrt{2 \pi}} \mathrm{e}^{-\frac{\left(x+a \sqrt{\frac{N}{N+1}}\right)^{2}}{2}} d x-\frac{2}{\sqrt{N+1}} \frac{1}{\sqrt{2 \pi}}\right) .
\end{aligned}
$$

Equivalent reasoning can be used to obtain the upper bound of (96). Thus, for finite $N$ one obtains

$2 \int_{\frac{1}{\sqrt{N+1}}}^{\frac{N-a \sqrt{N}+1}{\sqrt{N+1}}} x^{2} \frac{1}{\sqrt{2 \pi}} \mathrm{e}^{-\frac{\left(x+a \sqrt{\frac{N}{N+1}}\right)^{2}}{2}} d x+2 M \geq p_{\text {succ }} \geq 2 \int_{\frac{1}{\sqrt{N+1}}}^{\frac{N-a \sqrt{N}+1}{\sqrt{N+1}}} x^{2} \frac{1}{\sqrt{2 \pi}} \mathrm{e}^{-\frac{\left(x+a \sqrt{\frac{N}{N+1}}\right)^{2}}{2}} d x-2 M-\sum_{s=0}^{\frac{N-k}{2}}(2 s+1)^{2} \delta_{s}$,

where $M=\frac{2}{\sqrt{N+1}} \frac{1}{\sqrt{2 \pi}}$. This bound can be further refined. On the one hand, clearly

$$
\int_{\frac{1}{\sqrt{N+1}}}^{\frac{N-a \sqrt{N}+1}{\sqrt{N+1}}} x^{2} \frac{1}{\sqrt{2 \pi}} \mathrm{e}^{-\frac{\left(x+a \sqrt{\frac{N}{N+1}}\right)^{2}}{2}} d x+M \leq \int_{0}^{\infty} x^{2} \frac{1}{\sqrt{2 \pi}} \mathrm{e}^{-\frac{\left(x+a \sqrt{\frac{N}{N+1}}\right)^{2}}{2}} d x+M .
$$

On the other hand

$$
\begin{aligned}
& \int_{\frac{1}{\sqrt{N+1}}}^{\frac{N-a \sqrt{N}+1}{\sqrt{N+1}}} x^{2} \frac{1}{\sqrt{2 \pi}} \mathrm{e}^{-\frac{\left(x+a \sqrt{\frac{N}{N+1}}\right)^{2}}{2}} d x-M-\sum_{s=0}^{\frac{N-k}{2}}(2 s+1)^{2} \delta_{s}= \\
& \int_{0}^{\infty} x^{2} \frac{1}{\sqrt{2 \pi}} \mathrm{e}^{-\frac{\left(x+a \sqrt{\frac{N}{N+1}}\right)^{2}}{2}} d x-M-\sum_{s=0}^{\frac{N-k}{2}}(2 s+1)^{2} \delta_{s}-\int_{0}^{\frac{1}{\sqrt{N+1}}} x^{2} \frac{1}{\sqrt{2 \pi}} \mathrm{e}^{-\frac{\left(x+a \sqrt{\frac{N}{N+1}}\right)^{2}}{2}} d x-\int_{\frac{N-a \sqrt{N}+1}{\sqrt{N+1}}}^{\infty} x^{2} \frac{1}{\sqrt{2 \pi}} \mathrm{e}^{-\frac{\left(x+a \sqrt{\frac{N}{N+1}}\right)^{2}}{2}} \\
& \int_{0}^{\infty} x^{2} \frac{1}{\sqrt{2 \pi}} \mathrm{e}^{-\frac{\left(x+a \sqrt{\frac{N}{N+1}}\right)^{2}}{2}} d x-M-\sum_{s=0}^{\frac{N-k}{2}}(2 s+1)^{2} \delta_{s}-I_{1}-I_{2} .
\end{aligned}
$$

One has

$$
I_{1}=\int_{0}^{\frac{1}{\sqrt{N+1}}} x^{2} \frac{1}{\sqrt{2 \pi}} \mathrm{e}^{-\frac{\left(x+a \sqrt{\frac{N}{N+1}}\right)^{2}}{2}} d x \leq \int_{0}^{\frac{1}{\sqrt{N+1}}} x^{2} \frac{1}{\sqrt{2 \pi}} \mathrm{e}^{-\frac{x^{2}}{2}} d x \leq \int_{0}^{\frac{1}{\sqrt{N+1}}} \frac{1}{N+1} \frac{1}{\sqrt{2 \pi}} d x=(N+1)^{-\frac{3}{2}} \frac{1}{\sqrt{2 \pi}}
$$

and having definition of the complementary error function $\operatorname{erfc}(x):=1-\operatorname{erf}(x)$, where $\operatorname{erf}(x):=\frac{2}{\sqrt{\pi}} \int_{0}^{x} \mathrm{e}^{-t^{2}} d t$ is the error function we write

$$
\begin{array}{r}
I_{2}=\int_{\frac{N-a \sqrt{N}+1}{\sqrt{N+1}}}^{\infty} x^{2} \frac{1}{\sqrt{2 \pi}} \mathrm{e}^{-\frac{\left(x+a \sqrt{\frac{N}{N+1}}\right)^{2}}{2}} d x \leq \int_{\frac{N-a \sqrt{N}+1}{\sqrt{N+1}}}^{\infty} x^{2} \frac{1}{\sqrt{2 \pi}} \mathrm{e}^{-\frac{x^{2}}{2}} d x:=\int_{b}^{\infty} x^{2} \frac{1}{\sqrt{2 \pi}} \mathrm{e}^{-\frac{x^{2}}{2}} d x= \\
\frac{b \mathrm{e}^{-\frac{b^{2}}{2}}}{\sqrt{2 \pi}}+\frac{1}{2} \operatorname{erfc}\left(\frac{b}{\sqrt{2}}\right)=\frac{b \mathrm{e}^{-\frac{b^{2}}{2}}}{\sqrt{2 \pi}}+\frac{1}{\sqrt{\pi}} \int_{\frac{b}{\sqrt{2}}}^{\infty} \mathrm{e}^{-\frac{x^{2}}{2}} d x \leq \frac{b \mathrm{e}^{-\frac{b^{2}}{2}}}{\sqrt{2 \pi}}+\mathrm{e}^{-\frac{b^{2}}{2}},
\end{array}
$$

where in (129) we use Hoeffiding bound, i.e. $\int_{t}^{\infty} \mathrm{e}^{-c x^{2}} d x \leq \frac{\sqrt{\pi}}{c} \mathrm{e}^{-c^{2} t^{2}}$ and $b=\frac{N+1-a \sqrt{N}}{\sqrt{N+1}}=\sqrt{N+1}-a \sqrt{\frac{N}{N+1}}$. Furthermore

$$
\begin{gathered}
\frac{b \mathrm{e}^{-\frac{b^{2}}{2}}}{\sqrt{2 \pi}}+\mathrm{e}^{-\frac{b^{2}}{2}}=\left(\frac{\left(\sqrt{N+1}-a \sqrt{\frac{N}{N+1}}\right)}{\sqrt{2 \pi}}+1\right) \mathrm{e}^{-\frac{\left(\sqrt{N+1}-a \sqrt{\frac{N}{N+1}}\right)^{2}}{2}} \leq \\
\left(\frac{\left(\sqrt{N+1}-a \sqrt{\frac{N}{N+1}}\right)}{\sqrt{2 \pi}}+1\right) \mathrm{e}^{-\frac{(\sqrt{N+1}-1)^{2}}{2}} \leq\left(\frac{\sqrt{N+1}}{\sqrt{2 \pi}}+1\right) \mathrm{e}^{-\frac{(\sqrt{N+1}-1}{2}}{ }^{2}
\end{gathered}
$$


Finally, taking into account (94), we can for a given $N$ and $k=a \sqrt{N}, a \in(0,1)$ formulate the following lower and upper bounds for $p_{\text {succ }}$ :

$$
\begin{aligned}
& 2\left(\int_{0}^{\infty} x^{2} \frac{1}{\sqrt{2 \pi}} \mathrm{e}^{-\frac{\left(x+a \sqrt{\frac{N}{N+1}}\right)^{2}}{2}} d x+\frac{2}{\mathrm{e} \sqrt{N+1}} \frac{1}{\sqrt{2 \pi}}\right) \geq p_{\text {succ }} \geq \\
& 2\left(\int_{0}^{\infty} x^{2} \frac{1}{\sqrt{2 \pi}} \mathrm{e}^{-\frac{\left(x+a \sqrt{\frac{N}{N+1}}\right)^{2}}{2}} d x-\frac{2}{\mathrm{e} \sqrt{N+1}} \frac{1}{\sqrt{2 \pi}}-\left(\frac{\sqrt{N+1}}{\sqrt{2 \pi}}+1\right) \mathrm{e}^{-\frac{(\sqrt{N+1}-1)^{2}}{2}}-(N+1)^{-\frac{3}{2}} \frac{1}{\sqrt{2 \pi}}\right)+ \\
& -\frac{2}{N+1}\left(4 N^{-1 / 4}+11 \sqrt{N+1} \mathrm{e}^{-\frac{\left(N^{5 / 8}-1\right)^{2}}{N+1}}\right)
\end{aligned}
$$

\section{References}

[1] Ron M. Adin and Yuval Roichman. Enumeration of standard young tableaux, 2014. URL https://arxiv . org/abs/1408.4497.

[2] A. C. Aitken. Xxvi. - the monomial expansion of determinantal symmetric functions. Proceedings of the Royal Society of Edinburgh. Section A. Mathematical and Physical Sciences, 61(3):300-310, 1943. DOI: $10.1017 /$ S0080454100006312.

[3] Leonardo Banchi, Jason Pereira, Seth Lloyd, and Stefano Pirandola. Convex optimization of programmable quantum computers. npj Quantum Information, 6(1):42, May 2020. ISSN 2056-6387. DOI: 10.1038/s41534020-0268-2.

[4] Salman Beigi and Robert König. Simplified instantaneous non-local quantum computation with applications to position-based cryptography. New Journal of Physics, 13(9):093036, 2011. ISSN 1367-2630. DOI: $10.1088 / 1367-2630 / 13 / 9 / 093036$.

[5] Charles H. Bennett, Gilles Brassard, Claude Crépeau, Richard Jozsa, Asher Peres, and William K. Wootters. Teleporting an unknown quantum state via dual classical and Einstein-Podolsky-Rosen channels. Physical Review Letters, 70(13):1895-1899, March 1993. DOI: 10.1103/PhysRevLett.70.1895.

[6] Andrew C. Berry. The accuracy of the gaussian approximation to the sum of independent variates. Transactions of the American Mathematical Society, 49(1):122-136, 1941. DOI: https://doi.org/10.1090/S00029947-1941-0003498-3.

[7] Harry Buhrman, Łukasz Czekaj, Andrzej Grudka, Michał Horodecki, Paweł Horodecki, Marcin Markiewicz, Florian Speelman, and Sergii Strelchuk. Quantum communication complexity advantage implies violation of a Bell inequality. Proceedings of the National Academy of Sciences, 113(12):3191-3196, March 2016. ISSN 0027-8424, 1091-6490. DOI: 10.1073/pnas.1507647113.

[8] Giulio Chiribella and Daniel Ebler. Quantum speedup in the identification of cause-effect relations. Nature Communications, 10:1472, Apr 2019. DOI: 10.1038/s41467-019-09383-8.

[9] Matthias Christandl, Felix Leditzky, Christian Majenz, Graeme Smith, Florian Speelman, and Michael Walter. Asymptotic performance of port-based teleportation. Communications in Mathematical Physics, 381(1):379-451, Jan 2021. ISSN 1432-0916. DOI: 10.1007/s00220-020-03884-0.

[10] Carl-Gustav Esseen. On the liapunoff limit of error in the theory of probability. Arkiv för Matematik, Astronomi och Fysik A28, pages 1-19, 1942.

[11] Carl-Gustav Esseen. A moment inequality with an application to the central limit theorem. Scandinavian Actuarial Journal, 1956(2):160-170, 1956. DOI: 10.1080/03461238.1956.10414946.

[12] W. Fulton. Young Tableaux. With Applications to Representation Theory and Geometry. Cambridge University Press, New York, 1997.

[13] W. Fulton and J. Harris. Representation Theory - A first Course. Springer-Verlag, New York, 1991.

[14] Satoshi Ishizaka and Tohya Hiroshima. Asymptotic Teleportation Scheme as a Universal Programmable Quantum Processor. Physical Review Letters, 101(24):240501, December 2008. DOI: 10.1103/PhysRevLett.101.240501.

[15] Satoshi Ishizaka and Tohya Hiroshima. Quantum teleportation scheme by selecting one of multiple output ports. Physical Review A, 79(4):042306, April 2009. DOI: 10.1103/PhysRevA.79.042306.

[16] Kabgyun Jeong, Jaewan Kim, and Soojoon Lee. Generalization of port-based teleportation and controlled teleportation capability. Phys. Rev. A, 102:012414, Jul 2020. DOI: 10.1103/PhysRevA.102.012414. 
[17] Marek Mozrzymas, Michał Horodecki, and Michał Studziński. Structure and properties of the algebra of partially transposed permutation operators. Journal of Mathematical Physics, 55(3):032202, March 2014. ISSN 0022-2488, 1089-7658. DOI: 10.1063/1.4869027.

[18] Marek Mozrzymas, Michał Studziński, and Michał Horodecki. A simplified formalism of the algebra of partially transposed permutation operators with applications. Journal of Physics A Mathematical General, 51(12):125202, Mar 2018. DOI: 10.1088/1751-8121/aaad15.

[19] Marek Mozrzymas, Michał Studziński, Sergii Strelchuk, and Michał Horodecki. Optimal port-based teleportation. New Journal of Physics, 20(5):053006, May 2018. DOI: 10.1088/1367-2630/aab8e7.

[20] Marek Mozrzymas, Michał Studziński, and Piotr Kopszak. Optimal Multi-port-based Teleportation Schemes. Quantum, 5:477, June 2021. ISSN 2521-327X. DOI: 10.22331/q-2021-06-17-477.

[21] M. Murao, D. Jonathan, M. B. Plenio, and V. Vedral. Quantum telecloning and multiparticle entanglement. Phys. Rev. A, 59:156-161, Jan 1999. DOI: 10.1103/PhysRevA.59.156.

[22] M. A. Nielsen and Isaac L. Chuang. Programmable quantum gate arrays. Phys. Rev. Lett., 79:321-324, Jul 1997. DOI: 10.1103/PhysRevLett.79.321.

[23] Jason Pereira, Leonardo Banchi, and Stefano Pirandola. Characterising port-based teleportation as universal simulator of qubit channels. 54(20):205301, apr 2021. DOI: 10.1088/1751-8121/abe67a.

[24] Stefano Pirandola, Riccardo Laurenza, Cosmo Lupo, and Jason L. Pereira. Fundamental limits to quantum channel discrimination. npj Quantum Information, 5:50, Jun 2019. DOI: 10.1038/s41534-019-0162-y.

[25] Marco Túlio Quintino, Qingxiuxiong Dong, Atsushi Shimbo, Akihito Soeda, and Mio Murao. Reversing unknown quantum transformations: Universal quantum circuit for inverting general unitary operations. Phys. Rev. Lett., 123:210502, Nov 2019. DOI: 10.1103/PhysRevLett.123.210502.

[26] Jona Schulz. The optimal Berry-Esseen constant in the binomial case. PhD thesis, University of Trier, 2016. URL https://ubt.opus.hbz-nrw.de/opus45-ubtr/frontdoor/deliver/index/docId/ 732/file/Dissertation_Schulz.pdf.

[27] Michal Sedlák, Alessandro Bisio, and Mário Ziman. Optimal Probabilistic Storage and Retrieval of Unitary Channels. Phys. Rev. Lett., 122(17):170502, May 2019. DOI: 10.1103/PhysRevLett.122.170502.

[28] Sergii Strelchuk, Michał Horodecki, and Jonathan Oppenheim. Generalized Teleportation and Entanglement Recycling. Physical Review Letters, 110(1):010505, January 2013. DOI: 10.1103/PhysRevLett.110.010505.

[29] Michał Studziński, Sergii Strelchuk, Marek Mozrzymas, and Michał Horodecki. Port-based teleportation in arbitrary dimension. Scientific Reports, 7:10871, Sep 2017. DOI: 10.1038/s41598-017-10051-4.

[30] Michał Studziński, Marek Mozrzymas, Piotr Kopszak, and Michał Horodecki. Efficient multi-port teleportation schemes, 2020. URL https://arxiv.org/abs/2008.00984.

[31] Michał Studziński, Marek Mozrzymas, and Piotr Kopszak. Degradation of the resource state in port-based teleportation scheme, 2021. URL https://arxiv.org/abs/2105.14886. 